\title{
Dissipative effects on the superfluid-to-insulator transition in mixed-dimensional optical lattices
}

\author{
E. Malatsetxebarria \\ Centro de Física de Materiales (CFM), Centro Mixto CSIC-EHU, Paseo Manuel de Lardizabal 5, E-20018 San Sebastian, Spain \\ and Donostia International Physics Center (DIPC), Paseo Manuel de Lardizabal 4, E-20018 San Sebastian, Spain \\ Zi Cai and U. Schollwöck \\ Department of Physics and Arnold Sommerfeld Center for Theoretical Physics, Ludwig-Maximilians-Universität München, \\ D-80333 München, Germany \\ Miguel A. Cazalilla \\ Department of Physics, National Tsing Hua University, 101 Sec. 2, Kuang-Fu Road, Hsinchu, Taiwan \\ and Centro de Física de Materiales (CFM), Centro Mixto CSIC-EHU, \\ and Donostia International Physics Center (DIPC), Paseo Manuel de Lardizabal 4, E-20018 San Sebastian, Spain
}

(Received 21 May 2013; published 16 December 2013)

\begin{abstract}
We study the superfluid-to-Mott-insulator transition of a mixture of heavy bosons and light fermions loaded in an optical lattice. We focus on the effect of the light fermions on the dynamics of the heavy bosons. It is shown that when the lattice potential is sufficiently deep to confine the bosons to one dimension but allow the fermions to freely move in three dimensions (i.e., a mixed-dimensionality lattice), the fermions act as an ohmic bath for bosons, leading to screening and dissipation effects on the bosons. Using a perturbative renormalization-group analysis, it is shown that the fermion-induced dissipative effects have no appreciable impact on the transition from the superfluid-to-Mott-insulator state at integer filling. On the other hand, dissipative effects are found to be very important in the half-filled case near the critical point. In this case, in the presence of a finite incommensurability that destabilizes the Mott phase, the bosons can still be localized by virtue of dissipative effects.
\end{abstract}

DOI: 10.1103/PhysRevA.88.063630

PACS number(s): 67.85.Pq, 67.60.Fp, 05.30.Rt

\section{INTRODUCTION}

The interest in systems of interacting bosons and fermions has been a recurrent and central topic in the study of manybody problems. Many early studies were concerned with dilute solutions of ${ }^{3} \mathrm{He}$ in ${ }^{4} \mathrm{He}$ (see, e.g., [1] for a review) as well as with the problem of electrons coupled to phonons in solids (see, e.g., [2]). This research led to the understanding of important phenomena such as the polaron and Cooper pairing [2]. More recently, these concepts have reemerged in the context of ultracold atomic gases [3,4], where new types of interacting Bose-Fermi mixtures have been experimentally realized [5-16]. Indeed, such experiments with ultracold gases have made it possible to study and envision Bose-Fermi systems [17-36] that can exhibit very different properties from their condensed-matter counterparts.

So far, a lot of research has focused on understanding how interactions with the bosonic component of the mixture influence the properties of the fermions and, in particular, how the interactions mediated by the bosons can possibly induce fermion superfluidity (see, e.g., [3,31,36] and references therein). The complementary problem, namely, understanding how the properties of bosons are modified by their interactions with fermions in a mixture, has only recently attracted interest, which is especially motivated by a series of groundbreaking experiments with Bose-Fermi mixtures loaded in optical lattices $[8-11,15]$.

Within this setup, in recent years, a number of groups has addressed the problem of how the addition of fermions to a Bose gas in an optical lattice affects the phase transition from the superfluid to Mott insulator in the latter
[7,9-11,15,24,25,37]. Thus, experimental observations have been reported indicating that fermions effectively decrease the quantum coherence of the bosons, thus making it easier for the latter to become Mott insulating [7,9-11,15]. In the case of attractive interactions between bosons and fermions, this has been explained by a "self-trapping" effect: the bosons move in the lattice potential that effectively becomes deeper by the addition of fermions to which the bosons are attracted. This self-trapping would have the opposite effect in the case of repulsive boson-fermion interactions. However, in this case, the two components were found not to mix in a deep lattice [11].

In connection with the experiments referred to above, there has been some theoretical discussion on other, perhaps more subtle, effects of adding fermions to an interacting boson system $[24,25]$. These effects concern the physics of polarons, where one particle (in this case, the boson) is dressed by its interactions with a different species (the fermions). Thus, the boson-boson interactions are screened, become less repulsive. The bosons also undergo dissipative effects, which involve the creation of real particle-hole pairs and other kinds of low-energy excitations in the Fermi gas. Indeed, as discussed below, within a weak-coupling approach, the self-trapping effect arises at first order in the strength of the boson-fermion interactions, whereas the polaronic and dissipative effects arise at second order.

Going beyond mean-field theory, Yang [37] has studied the effect of the boson-fermion interaction on the superfluid (SF) to Mott-insulator (MI) transition in a three-dimensional (3D) Bose-Fermi mixture. He found that the properties of the transition at the particle-hole symmetric point (i.e., at 
the tip of the "Mott lobes") are modified and the transition becomes either a first-order transition or a different (i.e., not $X Y)$ second-order transition. However, in this work, we find that in a mixed-dimensionality system, the universality class of the transition (2D $X Y$ ) is not modified by the boson-fermion coupling for integer filling. The latter only introduces screening of the external periodic potential and the boson-boson interactions. Whereas the former tends to make the system less (more) superfluid for attractive (repulsive) boson-fermion interactions, the screening of the boson interactions always favors superfluidity. On the other hand, for a half-filled lattice, we find that the quantum phase transition from the SF to the charge-density wave (CDW) phase is modified by the presence of the fermions. However, the transition remains continuous and belongs to the $2 \mathrm{D} X Y$ universality class.

The outline of this article is as follows. In the following section, we introduce the basic model of a Bose-Fermi mixture that will be subsequently analyzed. There we also outline the derivation of its effective low-energy description. In Sec. III, we consider the effect of the Fermi gas on the Mott-insulator-to-superfluid transition of a Bose gas confined to one dimension [38-41]. The perturbative renormalization group is used to analyze the low-energy properties of the effective low-energy model, and the effect of the fermions on the superfluid-to-insulator quantum phase transition is studied. Depending on the lattice filling and the boson-boson interactions, the Mott insulator can be stabilized at integer or half-integer filling, while the effects of the Fermi gas on both transitions are very different. Thus, we have separated the discussion into two sections, i.e., Secs. III A and III B. Finally, in Sec. V, the main conclusions of this work are summarized.

\section{BASIC MODEL}

\section{A. Hamiltonian}

The system under study is an ultracold mixture of bosonic atoms (mass $m_{B}$ ) and single-species fermionic atoms (mass $m_{F}$ ) loaded in an optical lattice (see Fig. 1). The repulsive interaction between bosons is described by an interaction potential $v_{B B}\left(\mathbf{r}-\mathbf{r}^{\prime}\right)$. The latter can be either the LeeYang-Huang pseudopotential, which accounts for the $s$-wave scattering of ultracold atoms (as for alkali-metal or alkalineearth atoms), or a dipolar potential (as for chromium or polar bosonic molecules). Furthermore, fermions and bosons are assumed to interact only via a short-range potential, which is also described by the Lee-Yang-Huang pseudopotential. Interfermion interactions are negligible because, by the Pauli principle, the dominant scattering channel for single-species fermions is $p$ wave, which, away from resonances, is very weak at ultracold temperatures. The optical lattice potential $U_{B(F)}(\mathbf{r})=U_{0 \|}^{B(F)} \sin ^{2} k_{L} x+U_{0 \perp}^{B(F)}\left(\sin ^{2} k_{L} y+\sin ^{2} k_{L} z\right)$, where $k_{L}=2 \pi / \lambda_{L}$ and $\lambda_{L}$ is the laser wavelength. It is further assumed that $U_{0 \|}^{B} \ll U_{0 \perp}^{B}$, that is, the bosons move in a strongly anisotropic three-dimensional lattice. We further assume that the bosons are heavier (i.e., $m_{F} / m_{B} \ll 1$ ), which means that their motion along two directions (here, $y$ and $z$ ) is strongly suppressed beyond zero-point motion, thus effectively confining them to one dimension for at least the duration of the experiment. However, the fermions, being lighter, can hop

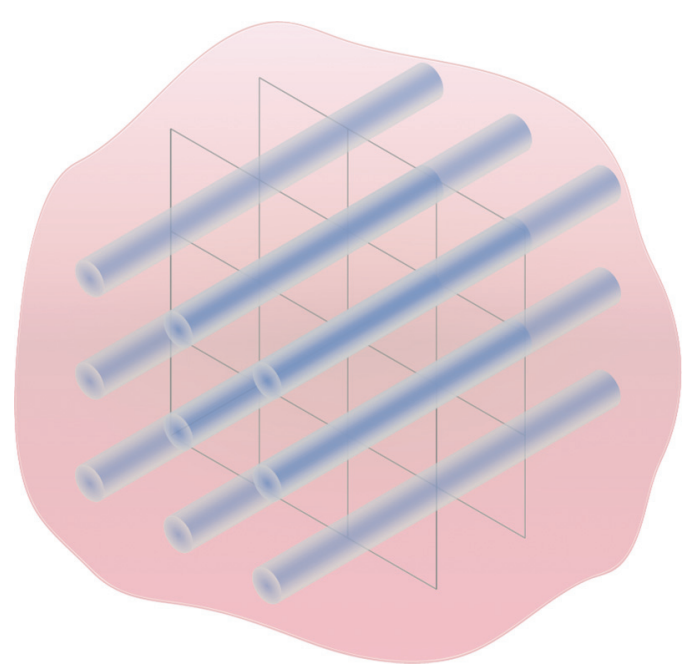

FIG. 1. (Color online) Schematic representation of the system studied in this work: A mixture of light fermions and heavy bosons loaded in an anisotropic optical lattice. As the bosons are assumed to be heavier, they are confined to one dimension by the lattice potential.

in all three dimensions, but the large laser intensity required to create the strong confining lattice potential for the bosons, which implies that $U_{0 \perp}^{F} \gg U_{0 \|}^{F}$, means the fermion dispersion will be anisotropic [see Eq. (6) below]. Thus, the Hamiltonian reads

$$
\begin{gathered}
\hat{H}=\hat{H}_{B}+\hat{H}_{F}+\hat{H}_{B F} \\
\hat{H}_{B}=\int d \mathbf{r}\left[\frac{\hbar^{2}}{2 m_{B}} \nabla \hat{\Psi}_{B}^{\dagger}(\mathbf{r}) \nabla \hat{\Psi}_{B}(\mathbf{r})+U_{B}(\mathbf{r}) \hat{\rho}_{B}(\mathbf{r})\right. \\
\left.+\frac{1}{2} \int d \mathbf{r}^{\prime} \hat{\rho}_{B}(\mathbf{r}) V_{B B}(\mathbf{r}-\mathbf{r}) \hat{\rho}_{B}\left(\mathbf{r}^{\prime}\right)\right] \\
\hat{H}_{F}=\int d \mathbf{r}\left[\frac{\hbar^{2}}{2 m_{F}} \nabla \hat{\Psi}_{F}^{\dagger}(\mathbf{r}) \nabla \hat{\Psi}_{F}(\mathbf{r})+U_{F}(\mathbf{r}) \hat{\rho}_{F}(\mathbf{r})\right] \\
\hat{H}_{B F}=g_{B F} \int d \mathbf{r} \hat{\rho}_{B}(\mathbf{r}) \hat{\rho}_{F}(\mathbf{r})
\end{gathered}
$$

where $\hat{\Psi}_{B(F)}(\mathbf{r})$ is the boson (fermion) field operator, which obeys $\left[\hat{\Psi}_{B}^{\dagger}(\mathbf{r}), \hat{\Psi}_{B}\left(\mathbf{r}^{\prime}\right)\right]=\delta(\mathbf{r}-\mathbf{r}) \quad\left[\left\{\hat{\Psi}_{F}^{\dagger}(\mathbf{r}), \hat{\Psi}_{F}\left(\mathbf{r}^{\prime}\right)\right\}=\delta(\mathbf{r}-\right.$ $\mathbf{r})],\left(\right.$ anti-)commuting otherwise; $\hat{\rho}_{B(F)}(\mathbf{r})=\hat{\Psi}_{B(F)}^{\dagger}(\mathbf{r}) \hat{\Psi}_{B(F)}(\mathbf{r})$ is the boson (fermion) density operator and $N_{B(F)}=$ $\int d \mathbf{r} \hat{\rho}_{B(F)}(\mathbf{r})$ is the boson (fermion) number operator. The boson-fermion interaction is parametrized by the coupling $g_{B F}=2 \pi \hbar^{2} a_{B F} / M_{B F}$, where $M_{B F}=m_{B} m_{F} /\left(m_{B}+m_{F}\right)$ is the reduced mass and $a_{B F}$ is the $s$-wave scattering length. Since we are interested in the ground-state phase diagram in the thermodynamic limit of the above system, we have neglected the harmonic trapping potential, which is also present in the experiments. Note that an implicit assumption of our analysis below is that the bosons and fermions are mixed. For short-range interactions between the bosons [i.e., for $\left.V_{B B}(\mathbf{r})=g_{B B} \delta(\mathbf{r})\right]$, the problem of the bosons and fermions forming a uniform mixture in the lattice geometry studied here has been previously considered in Ref. [32]. One conclusion of this work is that the uniform mixed phase in this Bose-Fermi system is always stable, provided the density of bosons and fermions is sufficiently high, for both attractive and repulsive interactions (see Ref. [32] for further details). 
The Hamiltonian introduced in Eqs. (1)-(4) contains too much information about energy scales in which we are not interested. Since our goal is to analyze the ground state and low-lying excitations of the system, we next derive an effective Hamiltonian that is much more appropriate toward this end. The first step is to project the Bose and Fermi fields onto the lowest Bloch band of the lattice potential. Thus, we expand $\hat{\Psi}_{B}(\mathbf{r}) \simeq \sum_{\mathbf{R}} w_{0}\left(\mathbf{r}_{\perp}-\mathbf{R}\right) \hat{\Psi}_{B \mathbf{R}}(x)$, where $w_{0}\left(\mathbf{r}_{\perp}-\mathbf{R}\right)$ are the Wannier orbitals describing particles localized around the site $\mathbf{R}=\frac{1}{2}(m, n) \lambda_{L}$ of a 2D (square) lattice. For the fermions, $\hat{\Psi}_{F}(\mathbf{r}) \simeq \sum_{\mathbf{k}} \varphi_{\mathbf{k}}(\mathbf{r}) \hat{f}_{\mathbf{k}}$, where $\varphi_{\mathbf{k}}(\mathbf{r})$ are the Bloch states of the lowest band. Note the differences in the treatment of the Bose and Fermi fields, which reflect their differences in mobility introduced by the conditions discussed above. Hence, upon neglecting terms coupling different lattice sites, the bosons are described by

$$
\begin{aligned}
\hat{H}_{B}= & \sum_{\mathbf{R}} \int d x\left[\frac{\hbar^{2}}{2 m_{B}}\left|\partial_{x} \hat{\Psi}_{B \mathbf{R}}(x)\right|^{2}+U_{B \|}(x) \hat{\rho}_{B \mathbf{R}}(x)\right] \\
& +\frac{1}{2} \sum_{\mathbf{R}} \int d x d x^{\prime} V_{B B}\left(x-x^{\prime}\right) \hat{\rho}_{B \mathbf{R}}(x) \hat{\rho}_{B \mathbf{R}}\left(x^{\prime}\right) .
\end{aligned}
$$

However, the fermions are described by

$$
\hat{H}_{F}=\sum_{\mathbf{k}} \epsilon(\mathbf{k}) \hat{f}_{\mathbf{k}}^{\dagger} \hat{f}_{\mathbf{k}}
$$

where the sum is over $\mathbf{k}$ belonging to the first Brillouin zone and $\epsilon(\mathbf{k})=\epsilon_{\|}(k)+\epsilon_{\perp}\left(\mathbf{k}_{\perp}\right) \simeq \frac{\hbar^{2} k^{2}}{2 m_{F}^{*}}-2 t_{\perp}\left(\cos k_{y} b_{0}+\right.$ $\cos k_{z} b_{0}$ ), where $b_{0}=\frac{\pi}{k_{L}}$ is the lattice parameter, and we have assumed that the periodic potential along the $x$ direction is so weak that it effectively amounts to a renormalization of the fermion mass. Finally, the boson-fermion interactions are described by

$$
\hat{H}_{B F}=g_{B F} \sum_{\mathbf{R}} \int d \mathbf{r}\left|w_{0}\left(\mathbf{r}_{\perp}-\mathbf{R}\right)\right|^{2} \hat{\rho}_{B \mathbf{R}}(x) \hat{\rho}_{F}(\mathbf{r}),
$$

where $\mathbf{r}=(x, y, z)=\left(x, \mathbf{r}_{\perp}\right)$. In the above expression, we have approximated the boson density operator $\hat{\rho}_{B}(\mathbf{r})=\hat{\rho}_{B}\left(x, \mathbf{r}_{\perp}\right) \simeq$ $\sum_{\mathbf{R}}\left|w_{0}\left(\mathbf{r}_{\perp}-\mathbf{R}\right)\right|^{2} \hat{\rho}_{B \mathbf{R}}(x)$.

\section{B. Integrating out the fermions}

The total Hamiltonian obtained upon projection onto the lowest Bloch band, $H=H_{B}+H_{F}+H_{B F}$, is still too complicated to solve. Since we are mainly interested in the low-temperature properties of the heavier bosons, which are much slower, a first step towards understanding the latter is to integrate out the fermionic degrees of freedom. To this end, we rely on the path-integral representation of the partition function $Z=\operatorname{Tr} e^{-\beta\left[H-\mu_{B} N_{B}-\mu_{F} N_{F}\right]}$ for the Hamiltonian, $H=$ $H_{B}+H_{F}+H_{F B}$, which allows us to write

$$
Z=\int\left[d \bar{\psi}_{B} d \psi_{B} d \bar{\psi}_{F} d \psi_{F}\right] e^{-S\left[\bar{\psi}_{B}, \psi_{B}, \bar{\psi}_{F}, \psi_{F}\right]},
$$

where

$$
\begin{gathered}
S=S_{B}+S_{F}+S_{B F}, \\
S_{B}=\sum_{\mathbf{R}} \int d x \int_{0}^{\hbar \beta} d \tau \bar{\psi}_{B \mathbf{R}}(x, \tau) \partial_{\tau} \psi_{B \mathbf{R}}(x, \tau) \\
\quad-\frac{\mu_{B}}{\hbar} \sum_{\mathbf{R}} \int d x \int_{0}^{\hbar \beta} d \tau\left|\psi_{B \mathbf{R}}(x, \tau)\right|^{2}+\int_{0}^{\hbar \beta} \frac{d \tau}{\hbar} H_{B}(\tau), \\
S_{F}=\sum_{\mathbf{k}} \int_{0}^{\hbar \beta} d \tau \bar{f}(x, \mathbf{k})\left[\partial_{\tau} f(\mathbf{k}, \tau)-\frac{\mu_{F}}{\hbar} f(\mathbf{k}, \tau)\right] \\
\quad+\frac{1}{\hbar} \int_{0}^{\hbar \beta} d \tau H_{F}(\tau), \\
S_{B F}=\frac{1}{\hbar} \int d \tau H_{B F}(\tau),
\end{gathered}
$$

where $\beta=\left(k_{B} T\right)^{-1}$ is the inverse of the absolute temperature and $k_{B}$ is Boltzmann's constant. Thus, the effective action for the bosons is defined by the following equation:

$$
e^{-S_{\text {eff }}\left[\bar{\psi}_{B}, \psi_{B}\right]}=\int[d \bar{f} d f] e^{-S_{B}-S_{F}-S_{B F}}=Z_{F}^{0} e^{-S_{B}}\left\langle e^{-S_{B F}}\right\rangle_{F},
$$

where $\langle\cdot\rangle_{F}=\operatorname{Tr} \hat{\rho}_{F} \ldots$ and $\hat{\rho}_{F}=Z_{F}^{-1} e^{-\beta\left(H_{F}-\mu N_{F}\right)}$, with $Z_{F}=\operatorname{Tr} e^{-\beta\left(H_{F}-\mu N_{F}\right)}$ being the noninteracting fermion partition function. To make further progress, we shall assume that the interaction between the bosons and the fermions is perturbatively small. Therefore, the above functional integral can be performed using the cumulant expansion, which yields

$$
\left\langle e^{-S_{B F}}\right\rangle_{F}=e^{-\left\langle S_{B F}\right\rangle+\frac{1}{2}\left\langle S_{B F}^{2}-\left\langle S_{B F}\right\rangle^{2}\right\rangle+\cdots} .
$$

The leading term is

$$
\begin{aligned}
\left\langle S_{B F}\right\rangle_{F}= & \frac{g_{B F}}{\hbar} \sum_{\mathbf{R}} \int_{0}^{\hbar \beta} d \tau \int d \mathbf{r}\left|w_{0}\left(\mathbf{r}_{\perp}-\mathbf{R}\right)\right|^{2} \\
& \times \rho_{B \mathbf{R}}(x, \tau) \rho_{F}^{0}(\mathbf{r}),
\end{aligned}
$$

where $\rho_{F}^{0}(\mathbf{r})=\left\langle\rho_{F}(\mathbf{r}, \tau)\right\rangle_{F}$ is the equilibrium density of the Fermi gas (in the absence of the bosons). Since $\rho_{F}^{0}(\mathbf{r})$ is periodic, (14) amounts to a correction to the periodic potential that the boson gas undergoes. The correction has the same sign as the coupling $g_{B F}$, which means that, e.g., for attractive boson-fermion interactions, the effective potential seen by the bosons is deepened by its (mean-field) interaction with the fermions. This effect has been termed self-trapping and has been studied both theoretically [15] and experimentally [9-11]. We shall not study it any further here. Instead, we focus on the second-order term, which leads to much more interesting physics. Neglecting the coupling between different sites $\mathbf{R}$ (i.e., terms where $\mathbf{R}^{\prime} \neq \mathbf{R}$ ) yields

$$
\begin{aligned}
-\frac{1}{2}\left\langle S_{B F}^{2}-\left\langle S_{B F}\right\rangle^{2}\right\rangle= & \frac{g_{B F}^{2}}{2 \hbar} \sum_{\mathbf{R}} \int d \mathbf{r} d \tau d \mathbf{r}^{\prime} d \tau^{\prime} \rho_{B \mathbf{R}}(x, \tau) \\
& \times \chi_{F}\left(x-x^{\prime}, \tau-\tau^{\prime}\right) \rho_{B \mathbf{R}}\left(x^{\prime}, \tau^{\prime}\right),
\end{aligned}
$$




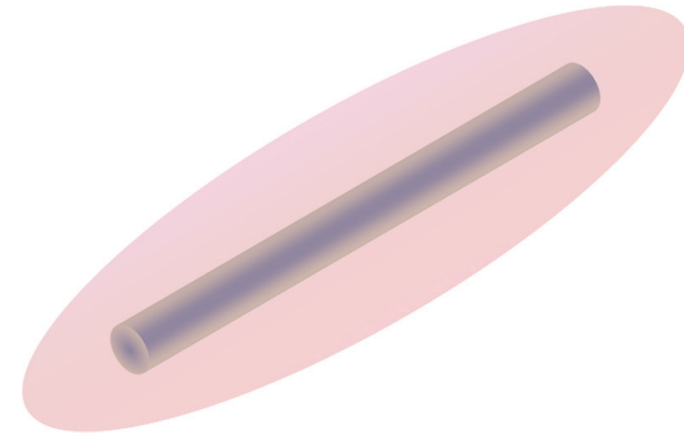

FIG. 2. (Color online) In the limit of strong one-dimensional (1D) confinement, the intertube coupling can be neglected and the system of Fig. 1 can be mapped to a single 1D tube immersed in a Fermi gas. The Fermi gas has two important effects: it introduces screening of the boson-boson interactions and it behaves as a dissipative bath that introduces quantum dissipation. Assuming that a periodic potential is applied longitudinally to the tube(s), which drives a transition from the superfluid to a Mott-insulating phase, we study the modification of the phase diagram due to these effects.

where $\mathbf{r}=\left(x, \mathbf{r}_{\perp}\right), \mathbf{r}^{\prime}=\left(x^{\prime}, \mathbf{r}_{\perp}^{\prime}\right)$. After defining $F_{0}\left(\mathbf{r}_{\perp}, \mathbf{r}_{\perp}\right)=$ $\left|w_{0}\left(\mathbf{r}_{\perp}\right) w_{0}\left(\mathbf{r}_{\perp}^{\prime}\right)\right|^{2}$, we introduce

$$
\begin{aligned}
\chi_{F}(x, \tau) & =\int d \mathbf{r}_{\perp} d \mathbf{r}_{\perp}^{\prime} F_{0}\left(\mathbf{r}_{\perp}, \mathbf{r}_{\perp}^{\prime}\right) \chi_{F}\left(\mathbf{r}, \mathbf{r}^{\prime}, \tau\right), \\
\chi_{F}\left(\mathbf{r}, \mathbf{r}^{\prime}, \tau\right) & =-\frac{1}{\hbar}\left\langle\delta \rho_{F}(\mathbf{r}, \tau) \delta \rho_{F}\left(\mathbf{r}^{\prime}, 0\right)\right\rangle_{F} .
\end{aligned}
$$

Thus, up to $O\left(g_{B F}^{2}\right)$, we obtain the following effective action for the bosons:

$$
\begin{aligned}
S_{\mathrm{eff}}\left[\psi_{B}^{*}, \psi_{B}\right]= & \sum_{R} S_{\mathrm{eff}, \mathbf{R}}, \\
S_{\mathrm{eff}, \mathbf{R}}= & \int_{0}^{\hbar \beta} d \tau \int d x \psi_{B \mathbf{R}}^{*}(x, \tau) \partial_{\tau} \psi_{B \mathbf{R}}(x, \tau) \\
& +\int_{0}^{\hbar \beta} d \tau \int d x \frac{\hbar}{2 m_{B}}\left|\partial_{x} \psi_{B \mathbf{R}}(x, \tau)\right|^{2} \\
& +\int_{0}^{\hbar \beta} \frac{d \tau}{\hbar} \int d x\left[\tilde{U}_{B \|}(x)-\mu_{B}\right]\left|\psi_{B \mathbf{R}}(x, \tau)\right|^{2} \\
& +\frac{1}{2} \int_{0}^{\hbar \beta} \frac{d \tau}{\hbar} \int d x d x^{\prime}\left|\psi_{B \mathbf{R}}(x, \tau)\right|^{2} \\
& \times V_{B B}\left(x-x^{\prime}\right)\left|\psi_{B \mathbf{R}}\left(x^{\prime}, \tau\right)\right|^{2} \\
& +\frac{g_{B F}^{2}}{2 \hbar} \int d x d \tau d x^{\prime} d \tau^{\prime}\left|\psi_{B \mathbf{R}}(x, \tau)\right|^{2} \\
& \times \chi_{F}\left(x, x^{\prime}, \tau-\tau^{\prime}\right)\left|\psi_{B \mathbf{R}}\left(x^{\prime}, \tau^{\prime}\right)\right|^{2},
\end{aligned}
$$

where $\quad \tilde{U}_{B \|}(x)=U_{B \|}(x)+g_{B F} \int d \mathbf{r}_{\perp}\left|w_{0}\left(\mathbf{r}_{\perp}\right)\right|^{2} \rho_{F}^{0}\left(x, \mathbf{r}_{\perp}\right)$. Note that we have thus reduced the problem to a set of one dimensional systems independently coupled to a fermionic bath, as shown in Fig. 2. Therefore, in what follows, we shall drop the lattice index $\mathbf{R}$ and study the phase diagram of a generic 1D system coupled to the fermionic bath. It is important to note that although a single 1D bosonic trap immersed in a 3D Fermi gas can be realized experimentally, assuming a deep lattice that tightly confines the bosons transversally allows us to neglect the coupling between different tubes. In this limit, the fermion-mediated intertube interactions can be neglected. Indeed, the density correlations in a Fermi gas are known to exhibit an oscillatory behavior, whose amplitude decays as a power law and gives rise to, e.g., the Friedel oscillations in the density. However, the effective intertube interactions are $g_{B F}^{2} \int d \mathbf{r}_{\perp}\left|w_{0}(\mathbf{r}-\mathbf{R})\right|^{2} \chi\left(x, x, \mathbf{r}_{\perp}, \mathbf{r}_{\perp}^{\prime}\right)\left|w_{0}\left(\mathbf{r}-\mathbf{R}^{\prime}\right)\right|^{2}, \quad$ which decay rapidly with the distance between the tubes $\mathbf{R}-\mathbf{R}^{\prime}$ since in a deep lattice the overlap of the Wannier functions $w_{0}(\mathbf{r}-\mathbf{R})$ is exponentially small. This means that any ordering tendencies mediated by such intertube interactions to states such as 3D Mott insulators or charge-density waves will take place at exponentially low temperatures. The latter are not experimentally reachable and therefore we can safely ignore such instabilities in the discussion which follows.

Furthermore, just like the fermions mediate interactions between the bosons, the converse is also true. Therefore, an important caveat when considering the applicability of the effective action, given by Eq. (17), is that we will not treat the bosons and the fermions on equal footing. Such a treatment would require us to account for the effect of the bosons on the fermionic component of the mixtures also, which may modify the density response $\chi_{F}\left(\mathbf{r}, \mathbf{r}^{\prime}, \tau\right)$. Nevertheless, below we shall assume that $\chi_{F}\left(\mathbf{r}, \mathbf{r}^{\prime}, \tau\right)$ is well described by the noninteracting limit where we take $g_{B F}=0$. Indeed, this assumption is qualitatively correct as long as the Fermi component of the mixture remains a Fermi liquid, which is reasonable given that the fermions are much lighter, interact with the bosons weakly, and therefore their behavior is dominated by the kinetic energy. However, strictly speaking, the bosons will mediate effective fermion-fermion interactions, which, at sufficiently low temperature, lead to a pairing instability of the Fermi gas. Since the gas contains a single species of fermions, such a pairing instability takes place in a high angular momentum wave (most likely, $p$ wave) and at relatively low temperatures compared to the Fermi energy $\mu_{F}$. Given that present cooling techniques in optical lattices cannot reach temperatures below a few percent of $\mu_{F}$, we can safely neglect this possibility. Other instabilities that can gap the fermion spectrum, such as a charge-density wave, occur at particular values of the lattice filling and/or lattice parameters and we will also neglect them in what follows.

\section{Low-energy effective theory}

In order to deal with the effective boson model in Eq. (17), we shall use the method of bosonization $[38,42]$. Thus, we first integrate out the high-energy density and phase fluctuations of the bosons and introduce two collective fields, $\theta(x)$ and $\phi(x)$, describing the phase and density fluctuations in each 1D system. In terms of these fields, the Bose field and density operators read

$$
\begin{gathered}
\Psi_{B}(x) \simeq \mathcal{A} \rho_{0}^{1 / 2} e^{i \theta(x)}, \\
\rho_{B}(x)=\Psi_{B}^{\dagger}(x) \Psi_{B}(x) \simeq \rho_{0}+\frac{1}{\pi} \partial_{x} \phi(x) \\
+\rho_{0} \sum_{m>0} \mathcal{B}_{m} \cos 2 m\left[\phi(x)+k_{F}^{B} x\right],
\end{gathered}
$$

where $\rho_{0}=N_{B} /(M L)$ is the linear density of bosons in each of the $M 1 \mathrm{D}$ systems of length $L$ of the lattice and $k_{F}^{B}=\pi \rho_{0}$. The amplitudes $\mathcal{A}$ and $\mathcal{B}_{m}$ depend on the microscopic details of 
the model and cannot be obtained using bosonization. Using the above expressions and retaining only the most relevant operators in the renormalization-group sense yields [38]

$$
\begin{gathered}
S_{B}[\phi]=S_{0}[\phi]+S_{u}[\phi], \\
S_{0}[\phi]=\frac{1}{2 \pi K} \int d x\left[\frac{1}{v}\left(\partial_{\tau} \phi\right)^{2}+v\left(\partial_{x} \phi\right)^{2}\right], \\
S_{u}[\phi]=-\frac{g_{u}}{\pi a_{0}^{2}} \int d \tau d x \cos \left[2 p \phi(x, \tau)+x \delta_{p}\right],
\end{gathered}
$$

where we have introduced the following notation: $v$ is the sound velocity of the $1 \mathrm{D}$ Bose gas, whereas $v K=v_{F}^{B}=$ $\hbar k_{F}^{B} / m_{B}$ and $K / v$ is proportional to the system compressibility; $a_{0} \approx \hbar v / \mu_{B}$ is a short-distance cutoff [38].

The bare dimensionless coupling of the term describing the periodic potential in the bosonization language is $g_{u}=$ $\tilde{U}_{0 \|}\left(\mathcal{B}_{p} \rho_{0} a_{0}^{2}\right) / 2 \hbar v$. The cosine term with $p=1$ describes the effect of the potential in the case of integer filling of the lattice, with $\delta_{p=1}=2\left(k_{L}-k_{F}^{B}\right)$ being a measure of the incommensurability of the system. However, near half filling, we must consider the $p=2$ term with $\delta_{p=2}=2 k_{L}-4 k_{F}^{B}$ as a measure of the incommensurability. In the half-filled case, the above effective Hamiltonian describes the transition from a Tomonaga-Luttinger liquid (TLL) to a fractional Mottinsulating state, which is also known as a charge-density wave (CDW). The stability of the CDW state requires smaller values of the Luttinger parameter $K$ [38] than those that are achievable in the Lieb-Liniger model [43] describing bosons interacting via a short-range potential in a 1D waveguide [44], for which the minimum value of $K$ is one [38]. Smaller values of $K$ are accessible either when the bosons possess dipolar moments $[38,45]$ or in the so-called super-Tonks regime [38,46,47].

The above action, given by Eq. (20), provides an effective description of the low-temperature properties of the boson system which includes (through the renormalization of the potential $\left.U_{B \|} \rightarrow \tilde{U}_{B \|}\right)$ the effect of the Fermi gas at the meanfield level. The dynamical effect of the fermions on the bosons is taken into account, to leading order in $g_{B F}$, by the last term in Eq. (17). However, since the dynamics of the (heavier) bosons described by (21) is much slower than that of the lighter fermions, some further simplifications of (15) are possible. First, we note (see Appendix B) that at $T=0$, the fermion density correlation function introduced above, $\chi_{F}\left(x, x^{\prime}, \tau\right)$, can be written as follows:

$$
\chi_{F}\left(x-x^{\prime}, \tau\right)=\int_{0}^{+\infty} \frac{d \omega}{\pi} e^{-\omega|\tau|} \operatorname{Im} \chi_{F}^{R}\left(x-x^{\prime}, \omega\right),
$$

where $\chi_{F}^{R}\left(x-x^{\prime}, \omega\right)$ is the retarded version of the same correlation function. We have also assumed, consistently with what was stated above, that the effect of the periodic potential can be neglected in the calculation of $\chi_{F}$ by taking $\chi_{F}\left(x, x^{\prime}, \omega\right)=\chi_{F}\left(x-x^{\prime}, \omega\right)$.

The above expression (23) allows us to treat the high-energy density fluctuations of the fermionic gas separately from the low-energy ones. This can be done by introducing the following response functions:

$$
\begin{aligned}
& \chi_{F}^{<}(x, \tau)=\int_{0}^{+\infty} \frac{d \omega}{\pi} g(\omega) e^{-\omega|\tau|} \operatorname{Im} \chi_{F}^{R}(x, \omega), \\
& \chi_{F}^{>}(x, \tau)=\int_{0}^{+\infty} \frac{d \omega}{\pi} g_{c}(\omega) e^{-\omega|\tau|} \operatorname{Im} \chi_{F}^{R}(x, \omega),
\end{aligned}
$$

where $g(\omega)$ is a frequency cutoff function, which can be chosen in various ways, as the result will be largely independent of this function; $g_{c}(\omega)=1-g(\omega)$. Below we use $g(\omega)=e^{-\omega \tau_{c}}$. In a Fermi gas, the bare cutoff energy $E_{c}=\frac{\hbar}{\tau_{c}}$ roughly corresponds to the Fermi energy, i.e., $E_{c} \approx E_{F}$. The separation into highand low-energy fluctuations allows us to treat the former using the adiabatic approximation. This is because high-energy (i.e., $\hbar \omega \gg E_{c} \sim E_{F}$ ) density fluctuations of the Fermi gas can adapt instantaneously to the slow dynamics of the much heavier bosons, as described by $\rho_{B}(x, \tau)$ [cf. Eq. (19)]. On the other hand, for low-energy density fluctuations of the Fermi gas $\left(\hbar \omega \ll E_{c} \sim E_{F}\right)$, adiabaticity breaks down and retardation effects become important. Thus,

$$
\begin{aligned}
\int & d x d \tau d x^{\prime} d \tau^{\prime} \rho_{B}(x, \tau) \chi_{F}^{>}\left(x-x^{\prime}, \tau-\tau^{\prime}\right) \rho_{B}\left(x^{\prime}, \tau^{\prime}\right) \\
= & \int d x d x^{\prime} d t d \tau \rho_{B}\left(x, \tau+\frac{t}{2}\right) \chi_{F}^{>}\left(x-x^{\prime}, t\right) \rho_{B}\left(x, \tau-\frac{t}{2}\right) \\
\simeq & \int d x d x^{\prime} d \tau \rho_{B}(x, \tau) \chi_{F}^{>}\left(x-x^{\prime}, \omega=0\right) \rho_{B}\left(x^{\prime}, \tau\right) \\
= & \int d x d x^{\prime} d \tau \rho_{B}(x, \tau) \chi_{F}\left(x-x^{\prime}, \omega=0\right) \rho_{B}\left(x^{\prime}, \tau\right) \\
& -\int d x d x^{\prime} d \tau \rho_{B}(x, \tau) \chi_{F}^{<}\left(x-x^{\prime}, \omega=0\right) \rho_{B}\left(x^{\prime}, \tau\right),
\end{aligned}
$$

where $\chi_{F}^{>}\left(x-x^{\prime}, \omega=0\right)=\int d t \chi_{F}^{>}\left(x-x^{\prime}, t\right)$ and similar definitions for $\chi_{F}^{<}\left(x-x^{\prime}, \omega=0\right)$ and $\chi_{F}\left(x-x^{\prime}, \omega=0\right)$. Therefore, the effective action describing the interactions between the bosons mediated by the Fermi gas takes the form

$$
\begin{aligned}
S_{\mathrm{eff}, B F}= & \frac{g_{B F}^{2}}{2 \hbar} \int d x d x^{\prime} d \tau \rho_{B}(x, \tau) \chi_{F}\left(x-x^{\prime}, \omega=0\right) \\
& \times \rho_{B}\left(x^{\prime}, \tau\right)+\frac{g_{B F}^{2}}{2 \hbar} \int d x d x^{\prime} d \tau d \tau^{\prime} \rho_{B}(x, \tau) \\
& \times \Gamma\left(x-x^{\prime}, \tau-\tau^{\prime}\right) \rho_{B}\left(x^{\prime}, \tau^{\prime}\right)
\end{aligned}
$$

where the dissipative kernel $\Gamma\left(x, x^{\prime}, \tau\right)$ is defined as

$$
\Gamma\left(x-x^{\prime}, \tau\right)=\chi_{F}^{<}(x, \tau)-\chi_{F}^{<}\left(x-x^{\prime}, \omega=0\right) \delta(\tau) .
$$

Note that by definition, $\int d \tau \Gamma\left(x-x^{\prime}, \tau\right)=0$. This kernel can be evaluated as follows. Since we assume the Fermi component of the mixture to be a Fermi liquid, we note that for the latter, $-\operatorname{Im} \chi_{F}^{R}\left(x-x^{\prime}, \omega\right) \propto \omega$ for $\omega \ll\left|\mu_{F}\right|[1]$. In the present system, the small $\omega$ limit of this function is obtained explicitly in Appendix B at $T=0$. It can be written as

$$
\operatorname{Im} \chi_{F}\left(x-x^{\prime}, \omega \ll \frac{\hbar}{\tau_{c}}\right)=-\pi D\left(x-x^{\prime}\right) \omega,
$$

where $D(x)$ is a positive function of $x$ which is computed in Appendix B. Introducing this expression into (24) yields

$$
\Gamma\left(x-x^{\prime}, \tau\right)=-\frac{D\left(x-x^{\prime}\right)}{\left(|\tau|+\tau_{c}\right)^{2}}
$$




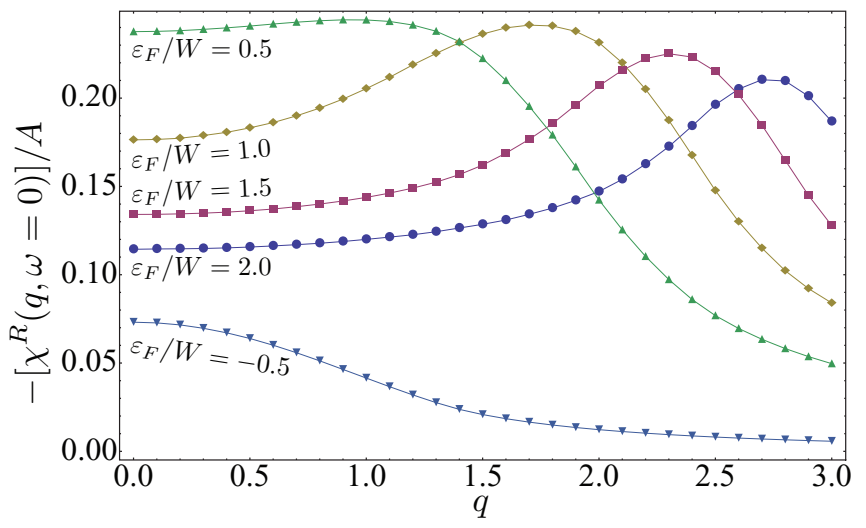

FIG. 3. (Color online) The static response function $\chi_{F}^{R}(q, \omega=$ $0) / A$ of the fermions for different values of the Fermi energy $\epsilon_{F}=$ $2.0,1.5,1.0,0.5,-0.5$ in units where $\frac{\hbar^{2}}{2 m}=1$ and $W=4 t_{\perp}=1$. See Appendix B for details of the calculation.

at $T=0$. Introducing the above expression into Eq. (27), we arrive at

$$
\begin{aligned}
S_{\mathrm{eff}, B F}= & \frac{g_{B F}^{2}}{2 \hbar} \int d x d x^{\prime} d \tau \rho_{B}(x, \tau) \chi_{F}\left(x-x^{\prime}, \omega=0\right) \\
& \times \rho_{B}\left(x^{\prime}, \tau\right)-\frac{g_{B F}^{2}}{2 \hbar} \int d x d x^{\prime} d \tau d \tau^{\prime} \rho_{B}(x, \tau) \\
& \times \frac{D\left(x-x^{\prime}\right)}{\left(\left|\tau-\tau^{\prime}\right|+\tau_{c}\right)^{2}} \rho_{B}\left(x^{\prime}, \tau^{\prime}\right) .
\end{aligned}
$$

The results of the model calculation described in Appendix B for the functions $D(q) / \hbar=-\operatorname{Im} \chi_{F}^{R}(q, \omega)$ [for $\omega \ll \frac{\hbar}{\tau_{c}}$ and the static response function $\left.\chi_{F}^{R}(q, \omega=0)\right]$ are displayed in Figs. 4 and 3. It can be seen that both functions are rather smooth (i.e., nonsingular) functions of the longitudinal wave vector $q$. This assumption will prove important below. Furthermore, for certain values of the lattice filling, which determine the Fermi energy $\epsilon_{F}$ (see Appendix B), $D(q)$ can be made negligible or zero for wide ranges of the wave number $q$ as $q \rightarrow 0$. This opens the possibility of tuning the strength of the dissipative

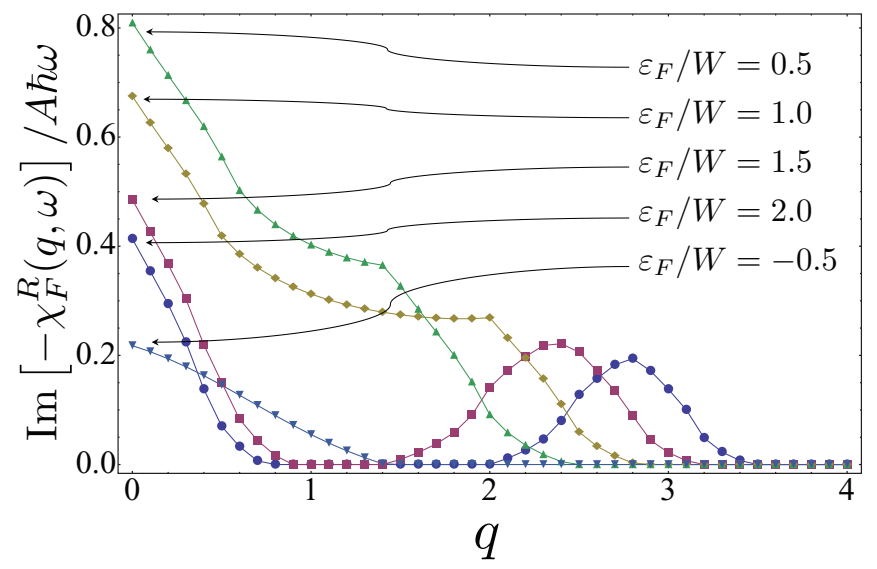

FIG. 4. (Color online) Imaginary part of the fermion response function divided by the excitation frequency $\omega$, for $\omega \rightarrow 0^{+}$, for different values of the Fermi energy $\epsilon_{F}=2.0,1.5,1.0,0.5,-0.5$. Units where $\frac{\hbar^{2}}{2 m_{F}}=1$ and $W=4 t_{\perp}=1$ have been used. See Appendix B for details of the calculation. effects by simply changing the fermion density. Note, however, that by strongly reducing the fermion density, the stability of the mixture may be jeopardized [32].

Thus we see that the boson interaction mediated by the Fermi gas consists, at low frequencies, of an instantaneous part (which stems from high-frequency density fluctuations of the Fermi gas) and a dissipative part, which takes the form of a retarded $\sim \frac{1}{\tau^{2}}$ interaction. The latter stems from the excitation by the motion of the bosons of real low-energy particle-hole pairs, which in a Fermi liquid yield the linear- $\omega$ behavior of the density response function (i.e., Landau damping). As discussed above, the instantaneous part of the interaction can be related to the static density response of the Fermi gas and leads to a renormalization of the sound velocity $v$ and Luttinger parameter $K$ describing the low-temperature properties of the $1 \mathrm{D}$ boson system. The renormalized parameters obey

$$
\frac{v\left(g_{B F}\right)}{K\left(g_{B F}\right)}=\frac{v\left(g_{B F}=0\right)}{K\left(g_{B F}=0\right)}+2 \frac{g_{B F}^{2}}{\hbar} \chi_{F}(q=0, \omega=0) .
$$

Furthermore, since the fermion-induced interaction is a density-density interaction [cf. first term in Eq. (31)], we have that [38]

$$
v\left(g_{B F}\right) K\left(g_{B F}\right)=v\left(g_{B F}=0\right) K\left(g_{B F}=0\right) .
$$

These equations describe, to lowest order in $g_{B F}$, the screening of the boson-boson interaction by the fermion gas, which leads to corrections to the parameters $K$ and $v$ in Eq. (21), which depend only on the boson-boson interaction.

Using the bosonization formula (19), we obtain the representation of the dissipative action in terms of the density field $\phi(x, \tau)$ :

$$
\begin{gathered}
\tilde{S}_{D}=S_{D}^{f}+S_{D}^{b}, \\
S_{D}^{f}=-\frac{\tilde{g}_{f D}}{\pi^{2}} \int d x d \tau d \tau^{\prime} \frac{\partial_{x} \phi(x, \tau) \partial_{x} \phi\left(x, \tau^{\prime}\right)}{\left(\left|\tau-\tau^{\prime}\right|+\tau_{c}\right)^{2}}, \\
S_{D}^{b}=-\frac{g_{b D}}{a_{0}} \int d x d \tau d \tau^{\prime} \frac{\cos 2\left[\phi(x, \tau)-\phi\left(x, \tau^{\prime}\right)\right]}{\left(\left|\tau-\tau^{\prime}\right|+\tau_{c}\right)^{2}} .
\end{gathered}
$$

In the derivation of the above perturbations to the Gaussian action, given by Eq. (21), we have retained only terms whose integrands are not oscillatory and are the leading terms in a gradient expansion. However, in the case of a half-filled lattice, the term

$$
S_{D}^{u}=-\frac{g_{u D}}{a_{0}} \int d x d \tau d \tau^{\prime} \frac{\cos 2\left[\phi(x, \tau)+\phi\left(x, \tau^{\prime}\right)\right]}{\left(\left|\tau-\tau^{\prime}\right|+\tau_{c}\right)^{2}}
$$

must also be taken into account. This dissipative umklapp interaction arises from the periodicity of the boson system. At half filling, $4 k_{F}^{B}=\frac{2 \pi}{b_{0}}$ is a reciprocal lattice wave number. In this regard, we must recall that in a periodic system, the (lattice) momentum along the $x$ direction is conserved modulo a reciprocal lattice wave number. Note that this term will also be generated by the renormalization-group flow from the product of the $S_{u}$ [cf. Eq. (22)] and $S_{D}^{b}$ [cf. Eq. (36)].

Furthermore, the bare dimensionless couplings are

$$
\begin{gathered}
\tilde{g}_{D}(0)=g_{B F}^{2} D(q=0), \\
g_{b D}(0)=2 g_{B F}^{2} \mathcal{B}_{1}^{2} \rho^{2} a_{0} D\left(q=2 k_{F}^{B}\right), \\
g_{u D}(0)=2 g_{B F}^{2} \mathcal{B}_{1}^{2} \rho^{2} a_{0} D\left(q=2 k_{F}^{B}\right) .
\end{gathered}
$$


In the above expressions, we have made explicit the dependence of the couplings on the cutoff scale $a_{0}$ through the parameter $\ell=\log \frac{a_{0}(\ell)}{a_{0}}$, that is, $a_{0}(\ell)=e^{\ell} a_{0}$, and thus $\ell=0$ corresponds to the scale of the bare cutoff $a_{0} \approx v \tau_{c}$, with $\tau_{c}$ being the short-time cutoff introduced earlier.

\section{RENORMALIZATION-GROUP ANALYSIS}

Physically, the renormalization group (RG) flow of a system describes its behavior as it is cooled down towards the absolute zero. The effect of temperature can be mimicked by decreasing the short-wavelength cutoff $\sim \frac{\hbar}{a_{0}}$ introduced to properly define the low-temperature effective model of the last section. As the absolute temperature decreases, the ground state is approached, and the couplings that define the effective low-energy theory of Eqs. (21), (22), (35), (36), etc. (i.e., $K$, $\left.v, g_{u}, g_{b D}, \ldots\right)$ must change accordingly in order to account for the reduction of the available excited states. Thus, the quantum phases of the system can be studied by analyzing the asymptotic behavior of the "flow" of these couplings in the limit where the cutoff tends to zero, that is, as the absolute temperature vanishes. In the perturbative approach to RG, the flow is described by a set of differential equations, whose solutions we study in this section.

Simple power-counting arguments show that $S_{D}^{f} \sim$ $\int d q d \omega q^{2}|\omega||\phi(q, \omega)|^{2}$ is an irrelevant perturbation in the renormalization-group sense. This is true provided $D(q=0)$ is not singular, which is indeed the case (see Fig. 4 and Appendix B). Indeed, this term alone leads to a momentumdependent broadening of the long-wavelength phonon excitations of the gapless phase of the model in Eq. (21). Therefore, in order to study the low-temperature properties of the model, it is justified to drop $S_{D}^{f}$, and therefore we shall next focus our attention on the second term in Eq. (34) and consider the effective model described by $S=S_{B}+S_{D}^{b}$, where $S_{B}$ is given by Eq. (21) and $S_{D}^{b}$ is given by Eq. (36). In the half-filled case, we also have to take into account $S_{D}^{u}$ given by Eq. (37). The resulting action contains only marginal and (potentially) relevant perturbations in the RG sense, which we shall analyze in this section. In what follows, we shall consider the cases of integer and half-integer lattice filling separately. The details of the perturbative derivation of the $\mathrm{RG}$ equations are given in Appendix C.

\section{A. Integer lattice filling}

To $O\left(g_{b D}, g_{u}^{2}\right)$, the flow equations in this case read

$$
\begin{gathered}
\frac{d g_{u}}{d \ell}=(2-K) g_{u}, \\
\frac{d g_{b D}}{d \ell}=(1-2 K) g_{b D}, \\
\frac{d K}{d \ell}=-\left(g_{u}^{2}+2 \pi g_{b D}\right) K^{2}, \\
\frac{d v}{d \ell}=-2 \pi g_{b D} K v .
\end{gathered}
$$

We neglect terms of $O\left(g_{b D}^{2}\right)$ or higher because $g_{b D}(0) \propto g_{B F}^{2}$, that is, $g_{b D}$ is already second order in the Bose-Fermi coupling, which is assumed to be small. For $g_{b D}=0$, the equations reduce to those of a pure $1 \mathrm{D}$ boson system in a commensurate potential first obtained by Haldane [40] (see also [38,39]); for $g_{u}=0$, the equations reduce to those derived in Ref. [48], which describe the quantum phase transition between a Tomonaga-Luttinger liquid and a dissipative insulator (DI).

The above equations show that near the SF-to-MI quantum critical point (corresponding to $K^{*}=2, g_{u}=0, g_{b D}=0$ ), the dissipative interaction is a highly irrelevant operator because $1-2 K \approx-3$. Thus, the most important effect of the Fermi component of the mixture is to introduce a renormalization of the periodic potential and the screening of the interactions, which leads to the renormalization of the Luttinger parameter $K$ and the sound velocity $v$ given by Eq. (32).

From the analysis of the RG equations, which implies that the dissipation is an irrelevant operator in the RG sense, we conclude that dissipative effects are weak in the MI phase where $g_{u}$ grows as the energy cutoff $\frac{\hbar v e^{-\ell}}{a_{0}}(\sim$ the absolute temperature) decreases. Thus, the dissipative term can be treated using perturbation theory and leads to a small (when compared to the excitation energy) broadening of the phonon excitations in the superfluid TLL phase. As for the excitations of the MI phase, which correspond to a "particle" (i.e., excess by one boson) or a "hole" (i.e., absence of a boson) propagating against the Mott-insulating background, the dissipative part of the interaction with the Fermi gas similarly introduces damping on their motion, which translates into the broadening of the excitation energy dispersion. Such enhancement of the excitation broadening can be measured by lattice modulation spectroscopy [41,49-51].

\section{B. Half-integer lattice filling}

In this case, and given that the initial conditions are the same for the $S_{D}^{b}$ and $S_{D}^{u}$ terms, we note that they can be combined into a single term, $S_{D}[\phi]=S_{D}^{b}[\phi]+S_{D}^{u}[\phi]$, which can be written as

$$
S_{D}[\phi]=\frac{g_{D}}{2 a_{0}} \int d x d \tau d \tau^{\prime} \frac{\left[\cos 2 \phi(x, \tau)-\cos 2 \phi\left(x, \tau^{\prime}\right)\right]^{2}}{\left(\left|\tau-\tau^{\prime}\right|+\tau_{c}\right)^{2}},
$$

where $g_{D}(0)=\frac{1}{2}\left[g_{b D}(0)+g_{u D}(0)\right]$. The RG flow equations for this system then read

$$
\begin{gathered}
\frac{d g_{u}}{d \ell}=(2-4 K) g_{u}+\pi g_{D}, \\
\frac{d g_{D}}{d \ell}=\left(1-2 K+4 g_{u}\right) g_{D}, \\
\frac{d K}{d \ell}=-\left(4 g_{u}^{2}+2 \pi g_{b D}\right) K^{2}, \\
\frac{d v}{d \ell}=-2 \pi g_{D} K v .
\end{gathered}
$$

These RG equations describe the flow in the vicinity of a quantum critical point located at $K^{*}=\frac{1}{2}, g_{u}^{*}=g_{D}^{*}=0$. Integrating them numerically, we obtain the phase diagram depicted in Fig. 5. Thus, we find that for a relatively weak boson-fermion coupling $\left|g_{B F}\right| / \mu_{B} \sim 10^{-2}$, the part of the phase diagram occupied by the SF Tomonaga-Luttinger liquid phase (TLL) shrinks considerably. The latter phase is identified by the RG flows for which both $g_{u}$ and $g_{D} \rightarrow 0$ as the cutoff $\hbar /\left(a_{0} e^{\ell}\right)$ is reduced to zero (i.e., for $\ell \rightarrow+\infty$ ), that is, as the absolute temperature is decreased. On the other hand, the 


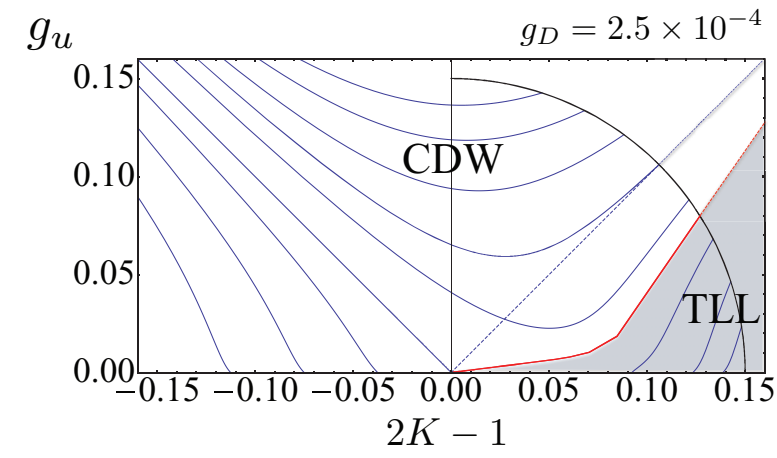

FIG. 5. (Color online) Phase diagram for the Tomonaga-Luttinger liquid (TLL) to charge-density wave (CDW) transition in the presence of a Fermi gas for $g_{D} \simeq 2.5 \times 10^{-4}$, which corresponds to $\left|g_{B F}\right| / \mu_{B} \sim 10^{-2}$ ( $\mu_{B}$ being the chemical potential of the bosons). $K$ is the Luttinger parameter of the bosons in the mixture [cf. Eq. (32)] and $g_{u} \propto U_{B \|}+O\left(g_{B F}\right)$, where $U_{B \|}$ is the external periodic potential. The shaded area is the TLL phase. The diagonal dashed line represents the TLL-CDW phase boundary in the absence of fermions. The curves in the diagram represent RG flows for the $K$ and $g_{u}$ couplings for a set of initial conditions lying on the quarter circle on the right. The flow proceeds from right to left as $K$ always decreases according to Eq. (48).

CDW phase is identified with those flows for which $g_{u} \sim 1$ at a certain value of $\ell^{*}$. However, it is also worth noticing that we have observed numerically (see Fig. 6) that especially close to the phase boundary (red curve in Fig. 5), $g_{u}\left(\ell^{*}\right) / g_{D}\left(\ell^{*}\right) \sim 1$, even if $g_{u}$ becomes of order one first in all cases studied. This means that even if the low-energy physics of this phase is dominated by the potential term $\propto g_{u}$, the dissipative effects are by no means negligible. It is interesting that this happens independently of how small the bare $g_{u}(0)$ is, and even in the limit $g_{u}(0) \rightarrow 0^{+}$. This is because, ultimately, the RG flow of $g_{u}(\ell)$ is controlled by the first term in Eq. (46), which leads to a much faster growth, although for small $g_{u}(0)$, the initial flow may be controlled by the second term in Eq. (46).

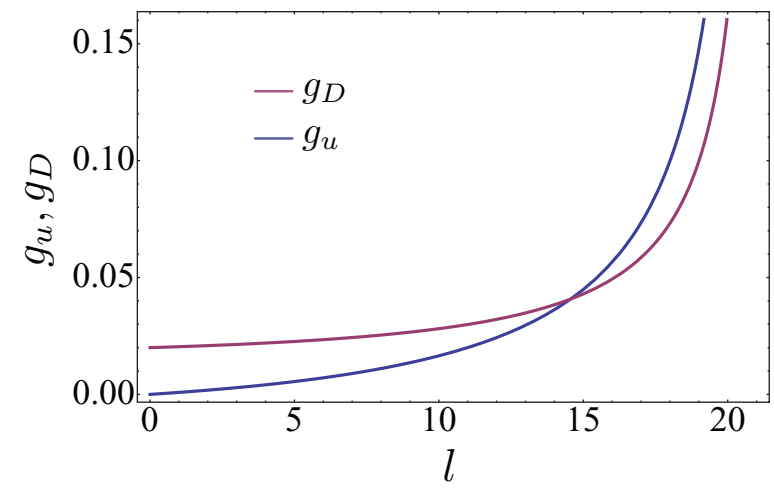

FIG. 6. (Color online) Runaway renormalization-group (RG) flow of the couplings $g_{u}$ (dark blue line), representing the periodic potential, and $g_{D}$ (light red line), representing the effect of the fermion-induced dissipation for $K \lesssim \frac{1}{2}$. We found that even for relatively small initial potential $g_{u}(0)$, the RG flow of $g_{u}(\ell)$ eventually overcomes the flow of $g_{D}(\ell)$ and becomes $g_{u}(\ell) \sim 1$ first. This means that the system localizes and becomes a Mott insulator. However, as this plot illustrates, the effect of $g_{D}$, i.e., the renormalized dissipative coupling, is not negligible.
The RG flow equations indicate that the quantum phase transition occurs at $K=1 / 2$, where the dissipation and periodic potential simultaneously become relevant, and the system is driven from superfluid to CDW Mott-insulating states. To study the interplay between the dissipation and interaction around the critical point, we adopt a variational self-consistent harmonic approximation (SCHA) by choosing a trial effective action of the form

$$
S_{v}[\phi]=\int \frac{d q d \omega}{(2 \pi)^{2}} G_{v}^{-1}(q, \omega) \phi^{*}(q, \omega) \phi(q, \omega),
$$

where we have defined the Green's function such that $G_{v}^{-1}(q, \omega)=\left[\frac{1}{2 \pi K}\left(\frac{\omega^{2}}{v_{s}}+v_{s} q^{2}\right)\right]+\left[\frac{\eta}{a_{0}}|\omega|+\frac{\Delta}{a_{0} \tau_{c}}\right]$ with the dimensionless self-consistent parameters $\eta$ and $\Delta$ that can be determined by the minimization of the variational free energy. A variational estimate $F_{\text {var }}$ of the true free energy $F$ can be obtained from Feynman's variational principle [39],

$$
F \leqslant F_{\text {var }}=F_{v}+\beta^{-1}\left\langle S-S_{v}\right\rangle_{v}
$$

Therefore, optimizing the trial free energy ( $\left.\delta F_{\mathrm{var}}\left[G_{v}\right] / \delta G_{v}=0\right)$, the parameters $\eta$ and $\Delta$ are found by solving the self-consistent equation above [see Eq. (D2)], so that (see Appendix D for further details)

$$
\begin{gathered}
\eta=\frac{8 g_{u}}{(2 \pi)^{2}} \alpha^{2}(\eta, \Delta, K), \\
\Delta=\frac{8\left(g_{u}+g_{D}\right)}{(2 \pi)^{2}} \alpha^{2}(\eta, \Delta, K),
\end{gathered}
$$

where we have introduced $\alpha(\eta, \Delta)=\left[\frac{\eta K \pi+2 \sqrt{K \pi \Delta}}{4}\right]^{2 K}$. The numerical solution of these equations for the gap $\Delta$ is shown in Fig. 7. It can be seen that the gap is enhanced for $K<$ $\frac{1}{2}$. This is expected because quantum dissipation is akin to classical friction, which hinders the motion of the particles and thus helps to stabilize the CDW Mott-insulating state. Note, however, that the SCHA erroneously yields a discontinuous transition for $K=\frac{1}{2}$. This is a well-known artifact of this approximation [39].

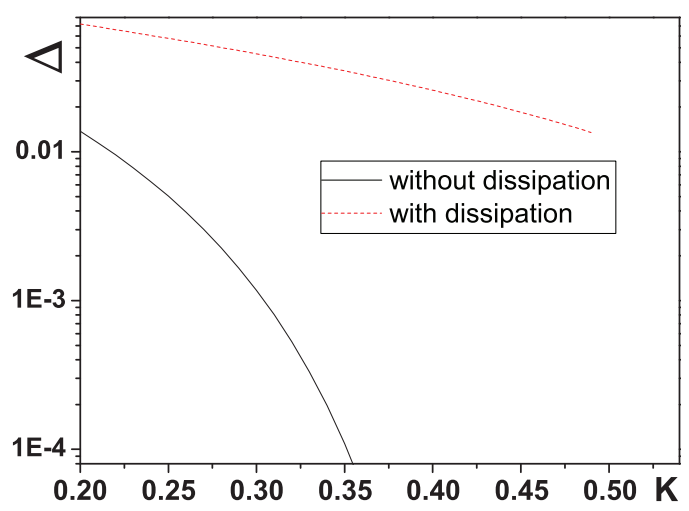

FIG. 7. (Color online) Mott gap $\Delta$ in the presence and absence of fermion-induced dissipation as obtained from the self-consistent harmonic approximation (SCHA; see Sec. III B for details). It can be seen that the dissipation greatly enhances the Mott gap by suppressing the quantum fluctuations of the bosons in the CDW Mott-insulating state. Note that the SCHA erroneously yields a discontinuous phase transition at the critical point $K^{*}=\frac{1}{2}$. This is a well-known artifact of this approximation [39]. 


\section{COMMENSURATE-INCOMMENSURATE TRANSITION IN THE PRESENCE OF DISSIPATION}

\section{A. Lattice near integer filling}

In this case, as for the TLL-to-MI transition, the effect of dissipation is rather weak. A way of understanding this is to stop the RG flow when $g_{u}(\ell) \sim 1$ and consider the sine-Gordon model at the Luther-Emery point where it maps to a 1D relativistic model of massive (Dirac) fermions [39,42]. Diagonalization of this model yields two bands separated by a gap: a filled "valence" band and an empty "conduction" band $[39,42]$. Tuning the chemical for the bosons amounts to introducing particles in the conduction band or holes in the valence band [42]. For small particle (hole) density, the system can be described as a Tonks-Girardeau gas [52] characterized by Luttinger parameter $K \simeq 1$. The dissipation being irrelevant for $K>K^{*}=\frac{1}{2}$, its effect on such a dilute liquid of particles (holes) is negligible as far as the ground-state properties are concerned (although it will lead to a small linewidth of the excitations, which is due to collisions between the bosons and the fermions). Thus, in particular, the exponents characterizing the commensurate-to-incommensurate (C-IC) transition are thus expected to remain unchanged and, therefore, the density of particles (or holes) $[38,42]$ will grow as $\sqrt{\mu-\mu_{c}}$, where $\mu_{c} \sim \Delta$, and where $\Delta$ is the MI gap.

\section{B. Lattice near half-integer filling}

For half-integer filling, the situation is very different, as was already pointed out in our discussion of the previous section. We can realize this by considering again the case where we take $g_{D}$ infinitesimally small, but $g_{u} \sim 1$. Applying the same reasoning used in the previous section, the sine-Gordon model $S_{b}[\phi]+S_{u}[\phi]$ in this case maps to a system of Dirac fermions describing the (fractionally charged) soliton and antisoliton excitations of the CDW state (configurations of the form 10101011010101 for the solitons and 101010010101 for the antisolitons). A dilute gas of such excitations can be described as a Luttinger gas with a parameter $K \simeq \frac{1}{4}<K^{*}=\frac{1}{2}$. Thus, the dissipative term $S_{D}[\phi]$ from Eq. (45) is a strongly relevant perturbation, which, as discussed in Ref. [48], leads to the localization of the system into a different phase, which we term dissipative insulator (DI). In this phase, the boson density $\left\langle\rho_{B}(x)\right\rangle$ exhibits long-range order [48] with a characteristic wave number equal to $4 \pi \rho_{0}$.

However, it is worth mentioning that as Fig. 6 demonstrates, the assumption that $g_{D}$ is infinitesimal when $g_{u} \sim 1$ is not representative of the the $\mathrm{RG}$ flow described in the previous section. Indeed, we found numerically that even in the case $g_{u}(0) \rightarrow$ $0, g_{D}\left(\ell^{*}\right) \lesssim g_{u}\left(\ell^{*}\right) \sim 1$ (see Fig. 6); in other words, the dissipation, although diverging less strongly than the periodic potential, is not a small perturbation on the CDW state. Thus, we expect that the dissipative term needs to be treated on equal footing with the potential term $\propto g_{u}$. The universality class of the commensurate-to-incommensurate transition is therefore expected to be different from the case of integer filling.

\section{CONCLUSIONS}

In conclusion, we have studied a model for a mixeddimensional Bose-Fermi mixture in an optical lattice, where the bosons are confined to one dimension, whereas the fermions are free to hop in three dimensions (albeit with renormalized dispersion). We have argued that this system is a realization of a $1 \mathrm{D}$ interacting Bose gas coupled to a dissipative bath of the ohmic type. In addition, the fermions also screen the boson-boson interactions. For integer filling of the boson lattice, we have found that the dominant effect of the fermions on the bosons is the screening of their interactions, as was also observed in mean-field studies of 3D dimensional optical lattices [25]. Thus, provided the so-called self-trapping effect can be subtracted or compensated, the screening of the boson interactions leads to an enhancement of the superfluid properties as the bosons become polarons with reduced effective interactions. In this case, dissipation effects only contribute to an increase in the linewidth of the excitations in both the superfluid and Mott-insulating phases, which could be detected by means of lattice modulation spectroscopy $[41,49,50]$.

On the other hand, the effect of the fermion-induced dissipation is much more severe when the bosons are close to a superfluid to CDW Mott-insulator transition, which happens at half-integer filling. In this case, the dissipative effects strongly hinder the motion of the bosons and help stabilize the CDW phase (cf. Fig. 5) as well as enhance the CDW gap (cf. Fig. 7). This effect leads to a dramatic suppression of the superfluid phase relative to the pure boson case, which can be observed as a reduction of the potential depth required for the bosons to localize in the CDW phase. The enhancement of the gap on the CDW side of the transition can also be probed using lattice modulation spectroscopy.

We have also studied the commensurate-incommensurate transition and argued that in the case of integer lattice filling, the fermion-induced dissipation is an irrelevant perturbation and, therefore, the universality class should not be altered. However, in the case of half-integer filling, the dissipation is relevant (but less than the external potential) and, therefore, we expect the universality class will be modified. This subject requires further study, but it will not be pursued here. The conclusions of this work are summarized in the schematic phase diagram of Fig. 8.

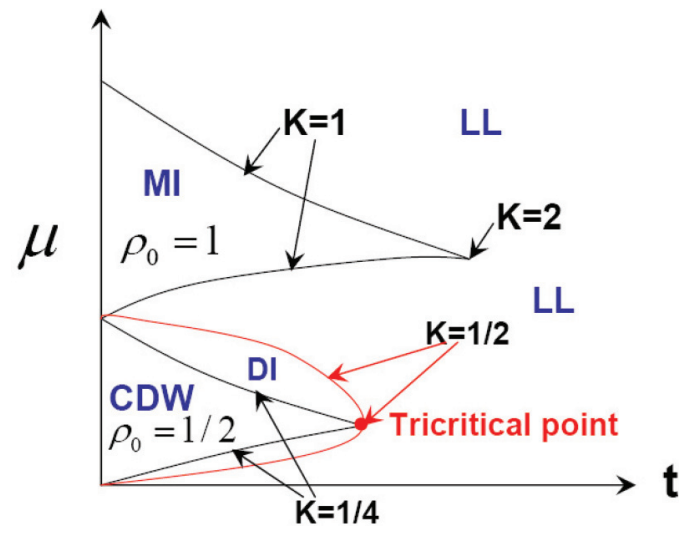

FIG. 8. (Color online) Schematic phase diagram of a system of heavy bosons confined to $1 \mathrm{D}$ and coupled to a dissipative bath of light fermions moving in 3D. $\mu$ is the chemical potential of the bosons, which controls the lattice filling; $t$ is related to the ratio of the mean kinetic to the mean interaction energy, which controls the Luttinger parameter $K$. 


\section{ACKNOWLEDGMENTS}

E.M. acknowledges support from the CSIC JAE-predoc program, co-financed by the European Science Foundation. E.M. and M.A.C. also acknowledge the support of the Basque Departamento de Educación, the UPV/EHU (Grant No. IT366-07), and the Spanish MINECO (Grant No. FIS201019609-CO2-02). U.S. and Z.C. acknowledge support from DFG through Project No. FOR801.

\section{APPENDIX A: RELATING $\chi_{F}^{R}(x, \omega)$ TO $\chi_{F}(x, \tau)$}

In this appendix, we will derive the identity that we used in the main text to relate the retarded density correlation function to its imaginary-time version at zero temperature. We shall first recall that the retarded correlation function is defined as

$$
\chi_{F}^{R}(x, t)=-\frac{i}{\hbar} \vartheta(t)\left\langle\left[\delta \rho_{F}(x, t) \delta \rho_{F}(0,0)\right]\right\rangle_{F},
$$

where $\delta \rho_{F}(x, t)=\int d \mathbf{r}_{\perp}\left|w_{0}\left(\mathbf{r}_{\perp}\right)\right|^{2} \delta \rho_{F}\left(x, \mathbf{r}_{\perp}, t\right), \delta \rho_{F}(x, t)=$ $e^{i H_{F} t / \hbar} \delta \rho_{F}(x) e^{-i H_{F} t / \hbar}$, and $\delta \rho_{F}(\mathbf{r})=\rho_{F}(\mathbf{r})-\rho_{F}^{0}(\mathbf{r})$. However, the imaginary-time correlation is defined as

$$
\chi_{F}(x, \tau)=-\frac{1}{\hbar}\left\langle\delta \rho_{F}(x, \tau) \delta \rho_{F}(0,0)\right\rangle_{F},
$$

where $\delta \rho_{F}(x, \tau)=e^{H_{F} \tau / \hbar} \delta \rho_{F}(x) e^{-H_{F} \tau / \hbar}$. By taking the Fourier transform of the spectral representation of (A1) and comparing it to the spectral representation of

$$
\chi_{F}\left(x, i \omega_{n}\right)=\int_{-\hbar \beta / 2}^{\hbar \beta / 2} d \tau \chi_{F}(x, \tau) e^{i \omega_{n} \tau},
$$

we arrive at the following relation:

$$
\begin{aligned}
\chi_{F}\left(x, i \omega_{n}\right) & =\int \frac{d \omega}{\pi} \frac{\operatorname{Im} \chi_{F}^{R}(x, \omega)}{\omega-i \omega_{n}} \\
& =\int_{0}^{+\infty} \frac{d \omega}{\pi}\left[\frac{\operatorname{Im} \chi_{F}^{R}(x, \omega)}{\omega-i \omega_{n}}+\frac{\operatorname{Im} \chi_{F}^{R}(x, \omega)}{\omega+i \omega_{n}}\right],
\end{aligned}
$$

where, in the derivation of the last expression, we have used that $\operatorname{Im} \chi_{F}(x,-\omega)=-\operatorname{Im} \chi_{F}(x, \omega)$. Hence, introducing the last expression in (A3), taking $\beta \rightarrow+\infty$, and performing the integral over $\omega_{n}$ with the help of Jordan's lemma, we arrive at the desired result:

$$
\chi_{F}(x, \tau)=\int_{0}^{+\infty} \frac{d \omega}{\pi} e^{-\omega|\tau|} \operatorname{Im} \chi_{F}^{R}(x, \omega) .
$$

\section{APPENDIX B: FERMION BATH RESPONSE FUNCTION}

Let us consider the Fourier transform of the density response of the Fermi gas at zero temperature, which, as we neglect the interactions induced by the bosons on the fermions, is just the Lindhard function. Recall that the Matsubara version of the latter is defined as $\chi_{F}\left(x, \mathbf{r}_{\perp}, \mathbf{r}_{\perp}^{\prime}, \tau\right)=-\frac{1}{\hbar}\left\langle\delta \rho_{F}\left(x, \mathbf{r}_{\perp}, \tau\right) \delta \rho_{F}\left(0, \mathbf{r}_{\perp}^{\prime}, 0\right)\right\rangle_{F}$, where $\delta \rho_{F}\left(x, \mathbf{r}_{\perp}\right)=\rho_{F}\left(x, \mathbf{r}_{\perp}\right)-\rho^{0}\left(x, \mathbf{r}_{\perp}\right)$, with $\rho_{F}\left(x, \mathbf{r}_{\perp}\right)=$ $\sum_{k, k^{\prime}, \mathbf{k}_{\perp}, \mathbf{k}_{\perp}^{\prime}} \varphi_{k, \mathbf{k}_{\perp}}^{*}\left(x, \mathbf{r}_{\perp}\right) \varphi_{k^{\prime}, \mathbf{k}_{\perp}^{\prime}}\left(x, \mathbf{r}_{\perp}\right) f_{k, \mathbf{k}_{\perp}}^{\dagger} f_{k^{\prime}, \mathbf{k}_{\perp}^{\prime}}$ being the density operator and $\rho^{0}\left(x, \mathbf{r}_{\perp}\right)=\left\langle\rho_{F}\left(x, \mathbf{r}_{\perp}\right)\right\rangle_{F}$ being the equilibrium density. We shall assume that the single-particle orbitals of the fermions are given by

$$
\begin{aligned}
\varphi_{k, \mathbf{k}_{\perp}}\left(x, \mathbf{r}_{\perp}\right) & =\varphi_{k}(x) \varphi_{\mathbf{k}_{\perp}}\left(\mathbf{r}_{\perp}\right) \\
& =\frac{1}{\sqrt{L M}} \sum_{\mathbf{R}} e^{i\left(k x+\mathbf{k}_{\perp} \cdot \mathbf{R}\right)} w_{0}^{F}\left(\mathbf{r}_{\perp}-\mathbf{R}\right),
\end{aligned}
$$

where $L$ is the (normalization) length in $1 \mathrm{D}, M$ is the number of lattice sites labeled by $\mathbf{R}=(n, m) b_{0}$ ( $b_{0}$ is the lattice parameter), and $w_{0}^{F}\left(\mathbf{r}_{\perp}\right)$ is the Wannier orbital for the fermions. In the above expression, we have assumed that the strength of the longitudinal potential in $1 \mathrm{D}$ is weak so that the Bloch orbitals $\varphi_{k}(x) \simeq \frac{e^{i k x}}{\sqrt{L}}$. Thus, we arrive at the following expression:

$$
\begin{aligned}
\chi_{F}\left(q, \mathbf{r}_{\perp}, \mathbf{r}_{\perp}^{\prime}, \omega\right)= & \int d \tau e^{i(\omega \tau-q x)} \chi_{F}\left(x, \mathbf{r}_{\perp}, \mathbf{r}_{\perp}^{\prime}, \tau\right) \\
= & \sum_{k, \mathbf{k}_{\perp}, \mathbf{k}_{\perp}^{\prime}} \frac{n_{k, \mathbf{k}_{\perp}}-n_{k+q, \mathbf{k}_{\perp}^{\prime}}}{i \hbar \omega-\epsilon\left(k+q, \mathbf{k}_{\perp}^{\prime}\right)+\epsilon\left(k, \mathbf{k}_{\perp}\right)} \\
& \times A_{\mathbf{k}_{\perp}, \mathbf{k}_{\perp}^{\prime}}\left(\mathbf{r}_{\perp}, \mathbf{r}_{\perp}^{\prime}\right),
\end{aligned}
$$

where the function $A_{\mathbf{k}_{\perp}, \mathbf{k}_{\perp}^{\prime}}\left(\mathbf{r}_{\perp}, \mathbf{r}_{\perp}^{\prime}\right)=$ $\varphi_{\mathbf{k}_{\perp}}^{*}\left(\mathbf{r}_{\perp}\right) \varphi_{\mathbf{k}_{\perp}}\left(\mathbf{r}_{\perp}^{\prime}\right) \varphi_{\mathbf{k}_{\perp}^{\prime}}\left(\mathbf{r}_{\perp}\right) \varphi_{\mathbf{k}_{\perp}^{\prime}}^{*}\left(\mathbf{r}_{\perp}^{\prime}\right)$. The single-particle dispersion of the fermions is

$\epsilon\left(k, \mathbf{k}_{\perp}\right)=\epsilon_{\|}(k)+\epsilon\left(\mathbf{k}_{\perp}\right)=\frac{\hbar^{2} k^{2}}{2 m_{F}^{*}}-2 t_{\perp}\left(\cos k_{y} b_{0}+\cos k_{z} b_{0}\right)$,

where we have assumed that the longitudinal dispersion is approximated by a quadratic dispersion characterized by an effective mass $m_{F}^{*} \approx m_{F}$ and transverse motion is described by a tight-binding dispersion characterized by a transverse hopping $t_{\perp}$.

Indeed, the response function in which we are interested is not the Lindhard function, given by (B2), but the following integral of it:

$$
\chi_{F}\left(q, \omega_{n}\right)=\int d \mathbf{r}_{\perp} d \mathbf{r}_{\perp}^{\prime} F_{0}\left(\mathbf{r}_{\perp}, \mathbf{r}_{\perp}^{\prime}\right) \chi\left(q, \mathbf{r}_{\perp}, \mathbf{r}_{\perp}^{\prime}, \omega_{n}\right),
$$

where $F_{0}\left(\mathbf{r}_{\perp}, \mathbf{r}_{\perp}^{\prime}\right)=\left|w_{0}\left(\mathbf{r}_{\perp}\right) w_{0}\left(\mathbf{r}_{\perp}\right)\right|^{2}$, and where $w_{0}(\mathbf{r})$ are the Wannier orbitals for the bosons in the lowest Bloch band. Thus, in order to compute (B4), we need to consider the following integral:

$$
\begin{aligned}
\int & d \mathbf{r}_{\perp} d \mathbf{r}_{\perp}^{\prime} F_{0}\left(\mathbf{r}_{\perp}, \mathbf{r}_{\perp}^{\prime}\right) A_{\mathbf{k}_{\perp}, \mathbf{k}_{\perp}^{\prime}}\left(\mathbf{r}_{\perp}, \mathbf{r}_{\perp}^{\prime}\right) \\
= & \left.\left.\left|\int d \mathbf{r}_{\perp} \varphi_{\mathbf{k}_{\perp}}^{*}\left(\mathbf{r}_{\perp}\right)\right| w_{0}\left(\mathbf{r}_{\perp}\right)\right|^{2} \varphi_{\mathbf{k}_{\perp}^{\prime}}\left(\mathbf{r}_{\perp}\right)\right|^{2} \\
= & \left.\left|\frac{1}{M} \sum_{\mathbf{R}, \mathbf{R}^{\prime}} e^{i\left(\mathbf{k}_{\perp} \cdot \mathbf{R}-\mathbf{k}_{\perp}^{\prime} \cdot \mathbf{R}^{\prime}\right)} \int d \mathbf{r}_{\perp}\right| w_{0}\left(\mathbf{r}_{\perp}\right)\right|^{2} \\
& \times\left.\left[w_{0}^{F}\left(\mathbf{r}_{\perp}-\mathbf{R}\right)\right]^{*} w_{0}^{F}\left(\mathbf{r}_{\perp}-\mathbf{R}^{\prime}\right)\right|^{2} \\
\simeq & \left.\left.\left|\frac{1}{M} \int d \mathbf{r}_{\perp}\right| w_{0}\left(\mathbf{r}_{\perp}\right)\right|^{2}\left|w_{0}^{F}\left(\mathbf{r}_{\perp}\right)\right|^{2}\right|^{2}=\frac{A}{M^{2}}
\end{aligned}
$$

where we have approximated $w_{0}\left(\mathbf{r}_{\perp}\right) \simeq e^{-\left|\mathbf{r}_{\perp}\right|^{2} / 2 \ell_{B \perp}^{2}} /\left(2 \pi \ell_{B \perp}^{2}\right)$ and $w_{0}^{F}\left(\mathbf{r}_{\perp}\right) \simeq e^{-\left|\mathbf{r}_{\perp}\right|^{2} / 2 \ell_{F \perp}^{2}} /\left(2 \pi \ell_{F \perp}^{2}\right)$ and assumed that $\ell_{B \perp} \ll \ell_{F \perp}$, so that we can neglect the overlap between the 
Wannier orbitals for $\mathbf{R} \neq \mathbf{R}^{\prime}$. In the above expression,

$$
A=\int d \mathbf{r}_{\perp}\left|w_{0}\left(\mathbf{r}_{\perp}\right)\right|^{2}\left|w_{0}^{F}\left(\mathbf{r}_{\perp}\right)\right|^{2}=\frac{1}{\pi^{2}\left(\ell_{F \perp}^{2}+\ell_{B \perp}^{2}\right)^{2}} .
$$

Hence,

$\chi_{F}\left(q, i \omega_{n}\right) \simeq \frac{A}{M^{2} L} \sum_{k, \mathbf{k}_{\perp}, \mathbf{k}_{\perp}^{\prime}} \frac{n_{k, \mathbf{k}_{\perp}}-n_{k+q, \mathbf{k}_{\perp}^{\prime}}}{i \hbar \omega_{n}-\epsilon\left(k+q, \mathbf{k}_{\perp}^{\prime}\right)+\epsilon\left(k, \mathbf{k}_{\perp}\right)}$.

Next, we take the thermodynamic limit, transform the sums over $k, \mathbf{k}_{\perp}, \mathbf{k}_{\perp}^{\prime}$ into integrals, and introduce the density of states of the 2D (square) lattice of tubes [53],

$$
\rho(\varepsilon)=\frac{2}{\pi^{2} W} K\left[\sqrt{1-\left(\frac{\varepsilon}{W}\right)^{2}}\right] \theta\left(W^{2}-\epsilon^{2}\right),
$$

where $K(z)$ denotes the complete elliptic integral of the first kind and $W=4 t_{\perp}$. Thus, the retarded response function [obtained from $\chi_{F}\left(q, i \omega_{n}\right)$ by means of analytic continuation where $i \omega_{n} \rightarrow \omega^{+}=\omega+i 0^{+}$] can be rewritten as follows:

$$
\begin{aligned}
\chi_{F}^{R}\left(q, \omega_{n}\right)= & A \int_{-W}^{+W} d \varepsilon d \varepsilon^{\prime} \rho(\varepsilon) \rho\left(\varepsilon^{\prime}\right) \\
& \times \int \frac{d k}{2 \pi} \frac{n_{k, \varepsilon}-n_{k+q, \varepsilon^{\prime}}}{\hbar \omega^{+}+\varepsilon-\varepsilon^{\prime}-\epsilon_{\|}(k+q)+\epsilon_{\|}(k)} \\
= & A \int_{-W}^{+W} d \varepsilon d \varepsilon^{\prime} \rho(\varepsilon) \rho\left(\varepsilon^{\prime}\right) \\
& \times \int \frac{d k}{2 \pi} n_{k, \varepsilon}\left[\frac{1}{\hbar \omega^{+}+\varepsilon-\varepsilon^{\prime}-\epsilon_{\|}(k+q)+\epsilon_{\|}(k)}\right. \\
& \left.+\frac{1}{-\hbar \omega^{+}+\varepsilon-\varepsilon^{\prime}+\epsilon_{\|}(k+q)-\epsilon_{\|}(k)}\right] \\
= & A \int_{-W}^{+W} d \varepsilon d \varepsilon^{\prime} \rho(\varepsilon) \rho\left(\varepsilon^{\prime}\right) \\
& \times \int_{\frac{d k}{2 \pi} n_{k, \varepsilon}\left[\frac{1}{\hbar \omega^{+}+\varepsilon-\varepsilon^{\prime}-\epsilon_{\|}(k+q)+\epsilon_{\|}(k)}\right.}^{(\mathrm{B} 9)} \\
& \left.+\left(\omega^{+} \rightarrow-\omega^{+}\right)\right] .
\end{aligned}
$$

At zero temperature, $n_{k, \varepsilon}=\theta\left(\epsilon_{F}-\epsilon-\epsilon_{\|}(k)\right)$, where $\epsilon_{F}=$ $\mu_{F}(T=0)$ is the Fermi energy (note that $\epsilon_{F}>-W$, otherwise there will be no fermions in the mixture).

Let us first consider (minus) the imaginary part of $\chi_{F}^{R}(q, \omega)$ :

$$
\begin{aligned}
\operatorname{Im}\left[-\chi_{F}^{R}(q, \omega)\right]= & \frac{A}{2} \int_{-W}^{+W} d \varepsilon \int d k \theta\left(\epsilon_{F}-\epsilon-\frac{\hbar^{2} k^{2}}{2 m_{F}^{*}}\right) \\
& \times \rho(\varepsilon)\left[\rho\left(\hbar \omega+\varepsilon-\frac{\hbar^{2} q^{2}}{2 m_{F}^{*}}-\frac{\hbar^{2} k q}{m_{F}^{*}}\right)\right. \\
& \left.-\rho\left(\hbar \omega-\varepsilon+\frac{\hbar^{2} q^{2}}{2 m_{F}^{*}}+\frac{\hbar^{2} k q}{m_{F}^{*}}\right)\right],
\end{aligned}
$$

where we have set $\epsilon_{\|}(k+q)-\epsilon_{\|}(k)=\frac{\hbar^{2} q^{2}}{2 m_{F}^{*}}+\frac{\hbar^{2} k q}{m_{F}^{*}}$. The above expression can be used to obtain the (imaginary part of the) response for arbitrary $\omega$. However, we are only interested in the regime of small $\omega$, for which we can expand $\rho(\hbar \omega \pm E(k, q, \varepsilon))=\rho(E(k, q, \varepsilon)) \pm \rho^{\prime}(E(k, q, \varepsilon)) \hbar \omega+$ .. [where $\left.E(k, q, \varepsilon)=\varepsilon-\frac{\hbar^{2}}{2 m_{F}^{*}}\left(q^{2}+2 k q\right)\right]$ and, therefore, to lowest order in $\omega$,

$$
\begin{aligned}
\operatorname{Im}\left[-\chi_{F}^{R}(q, \omega)\right] \simeq & A \hbar \omega \int_{-W}^{+W} d \varepsilon \int d k \rho(\varepsilon) \\
& \times \rho^{\prime}\left(\varepsilon-\frac{\hbar^{2}}{2 m_{F}^{*}}\left(q^{2}+2 k q\right)\right) \\
& \times \theta\left(\epsilon_{F}-\varepsilon-\frac{\hbar^{2} k^{2}}{2 m_{F}^{*}}\right) .
\end{aligned}
$$

In order to perform the integration over $k$, we define, from the constraints imposed by the Heaviside step function in Eq. (B12), $k_{F}(\varepsilon)=\sqrt{\frac{2 m_{F}^{*}}{\hbar^{2}}\left(\epsilon_{F}-\varepsilon\right)}$ for $\varepsilon<\epsilon_{F}$, and note that

$$
\begin{aligned}
& \int_{-k_{F}(\varepsilon)}^{+k_{F}(\varepsilon)} d k \partial_{\varepsilon} \rho\left(\varepsilon-\frac{\hbar^{2}}{2 m_{F}^{*}}\left(q^{2}+2 k q\right)\right) \\
& =-\frac{m_{F}^{*}}{\hbar^{2} q} \int_{-k_{F}(\varepsilon)}^{+k_{F}(\varepsilon)} d k \partial_{k} \rho\left(\varepsilon-\frac{\hbar^{2}}{2 m_{F}^{*}}\left(q^{2}+2 k q\right)\right) \\
& =-\frac{m_{F}^{*}}{\hbar^{2} q}\left(\rho\left\{\varepsilon-\frac{\hbar^{2}}{2 m_{F}^{*}}\left[q^{2}+2 k_{F}(\varepsilon) q\right]\right\}\right. \\
& \left.-\rho\left\{\varepsilon-\frac{\hbar^{2}}{2 m_{F}^{*}}\left[q^{2}-2 k_{F}(\varepsilon) q\right]\right\}\right) .
\end{aligned}
$$

Thus, the expression is simplified and only the integration over $\varepsilon$ remains:

$$
\begin{aligned}
\operatorname{Im}\left[-\chi^{R}(q, \omega)\right] \simeq & A \hbar \omega\left(-\frac{m_{F}^{*}}{\hbar^{2} q}\right) \int_{-W}^{+W} d \varepsilon \theta\left(\epsilon_{F}-\varepsilon\right) \rho(\varepsilon) \\
& \times\left(\rho\left\{\varepsilon-\frac{\hbar^{2}}{2 m_{F}^{*}}\left[q^{2}+2 k_{F}(\varepsilon) q\right]\right\}\right. \\
& \left.-\rho\left\{\varepsilon-\frac{\hbar^{2}}{2 m_{F}^{*}}\left[q^{2}-2 k_{F}(\varepsilon) q\right]\right\}\right) .
\end{aligned}
$$

This expression can be numerically evaluated (cf. Fig. 4). However, for $q \rightarrow 0$, further analytical progress is possible by noting that $\rho(\varepsilon) \rho^{\prime}(\varepsilon)=\frac{1}{2} d[\rho(\varepsilon)]^{2} / d \varepsilon$ and, hence,

$$
\operatorname{Im}\left[-\chi_{F}^{R}(q \rightarrow 0, \omega)\right] \simeq A \hbar \omega \int_{-W}^{\min \left\{+W, \epsilon_{F}\right\}} d \varepsilon k_{F}(\varepsilon) \frac{d[\rho(\varepsilon)]^{2}}{d \varepsilon} .
$$

From which, upon integration by parts, we obtain

$$
\begin{aligned}
\operatorname{Im}\left[-\chi_{F}^{R}(q \rightarrow 0, \omega)\right] \simeq & A \hbar \omega\left\{-[\rho(-W)]^{2} k_{F}(-W)\right. \\
& +\left[\rho\left(\min \left\{W, \epsilon_{F}\right\}\right)\right]^{2} k_{F}\left(\min \left\{W, \epsilon_{F}\right\}\right) \\
& \left.+\frac{m_{F}^{*}}{\hbar^{2}} \int_{-W}^{\min \left\{W, \epsilon_{F}\right\}} d \varepsilon \frac{[\rho(\varepsilon)]^{2}}{k_{F}(\varepsilon)}\right\} \\
= & A \hbar \omega \frac{m_{F}^{*}}{\hbar^{2}} \int_{-W}^{\min \left\{+W, \epsilon_{F}\right\}} d \varepsilon \frac{[\rho(\varepsilon)]^{2}}{k_{F}(\varepsilon)} .
\end{aligned}
$$


Hence, by direct numerical evaluation of the above expression, we see that it is not singular, which implies that the $q \sim 0$ term [denoted $S_{D}^{f}$ in Eq. (35)] can be neglected. In general, using Eq. (B12) to evaluate $\operatorname{Im}\left[-\chi_{F}^{R}(q, \omega)\right]$ for finite $q$, we find it is also a nonsingular function of $q$ in the neighborhood of $q=2 k_{F}^{B}=2 \pi \rho^{0}$. The results of a numerical evaluation of the integrals in Eqs. (B12) and (B15) are displayed in Fig. 4.

Finally, the real part of the response function is given by

$$
\begin{aligned}
\operatorname{Re}\left[\chi_{F}^{R}(q, \omega)\right]= & A \int_{-\infty}^{\infty} d \varepsilon d \varepsilon^{\prime} \rho(\varepsilon) \rho\left(\varepsilon^{\prime}\right) \int \frac{d k}{2 \pi} n_{k, \varepsilon} P\left[\frac{1}{\hbar \omega+\varepsilon-\varepsilon^{\prime}-\epsilon_{\|}(k+q)+\epsilon_{\|}(k)}+(\omega \rightarrow-\omega)\right] \\
= & A \int_{-W}^{\min \left[W, \epsilon_{F}\right]} d \varepsilon \rho(\varepsilon) \int \frac{d k}{2 \pi} \theta\left(\epsilon_{F}-\varepsilon-\frac{\hbar^{2} k^{2}}{2 m_{F}^{*}}\right) \\
& \times \int_{-W}^{\min \left[W, \epsilon_{F}\right]} d \varepsilon^{\prime} P\left[\frac{\rho\left(\varepsilon^{\prime}\right)}{\hbar \omega+\varepsilon-\varepsilon^{\prime}-\epsilon_{\|}(k+q)+\epsilon_{\|}(k)}+(\omega \rightarrow-\omega)\right] \\
= & A \int_{-W}^{\min \left[W, \epsilon_{F}\right]} d \varepsilon \rho(\varepsilon) \int_{-k_{F}(\varepsilon)}^{k_{F}(\varepsilon)} \frac{d k}{2 \pi} \int_{-W}^{\min \left[W, \epsilon_{F}\right]} d \varepsilon^{\prime} P\left[\frac{\rho\left(\varepsilon^{\prime}\right)}{E-\varepsilon^{\prime}}+(\omega \rightarrow-\omega)\right],
\end{aligned}
$$

where we have introduced $E=\hbar \omega+\varepsilon-\epsilon_{\|}(k+q)+\epsilon_{\|}(k)$. Furthermore, by using the well-known Kramers-Kronig relations that connect the real and imaginary part of any complex function which is analytic in the upper half plane,

$$
\operatorname{Re}\left[G^{R}(\mathbf{R}=0, \varepsilon)\right]=-P \int_{-\infty}^{\infty} \frac{d \varepsilon^{\prime}}{\pi} \frac{\operatorname{Im}\left[G^{R}\left(\mathbf{R}=\mathbf{0}, \varepsilon^{\prime}\right)\right]}{\varepsilon-\varepsilon^{\prime}},
$$

we have that

$$
P \int d \varepsilon^{\prime} \frac{\rho\left(\varepsilon^{\prime}\right)}{E-\varepsilon^{\prime}}=P \int d \varepsilon^{\prime} \frac{(-1 / \pi) \operatorname{Im}\left[G^{R}\left(\mathbf{R}=\mathbf{0}, \varepsilon^{\prime}\right)\right]}{E-\varepsilon^{\prime}}=\operatorname{Re}\left[G^{R}(\mathbf{R}=\mathbf{0}, E)\right] .
$$

Then, we can rewrite Eq. (B16), so that

$$
\begin{aligned}
\operatorname{Re}\left[\chi_{F}^{R}(q, \omega)\right] & =A \int_{-W}^{\min \left[W, \epsilon_{F}\right]} d \varepsilon \rho(\varepsilon) \int_{-k_{F}(\varepsilon)}^{k_{F}(\varepsilon)} \frac{d k}{2 \pi}\left\{\operatorname{Re}\left[G^{R}(\mathbf{R}=\mathbf{0}, E)\right]+(\omega \rightarrow-\omega)\right\} \\
& =\frac{A}{2 \pi} \int_{-W}^{\min \left\{W, \epsilon_{F}\right\}} d \varepsilon \rho(\varepsilon) \int_{-k_{F}(\varepsilon)}^{+k_{F}(\varepsilon)} d k\left[g\left(\hbar \omega+\varepsilon-\frac{\hbar^{2} q^{2}}{2 m_{F}^{*}}-\frac{\hbar^{2} k q}{m_{F}^{*}}\right)+g\left(-\hbar \omega+\varepsilon-\frac{\hbar^{2} q^{2}}{2 m_{F}^{*}}-\frac{\hbar^{2} k q}{m_{F}^{*}}\right)\right],
\end{aligned}
$$

where $g(\varepsilon)=-\operatorname{Re}\left[G^{R}(\mathbf{R}=\mathbf{0}, \varepsilon)\right]$ is the Hilbert transform of the density of states in a $2 \mathrm{D}$ square lattice modeled by a tightbinding approximation [53]:

$$
g(\varepsilon)=P \int d \varepsilon^{\prime} \frac{\rho\left(\varepsilon^{\prime}\right)}{\varepsilon^{\prime}-\varepsilon}= \begin{cases}-\frac{2}{\pi \varepsilon} \mathcal{K}\left(\frac{\varepsilon}{W}\right) & \text { for }|\varepsilon| \geqslant W \\ -\frac{\operatorname{sgn}(\varepsilon)}{\pi W} \mathcal{K}\left(\frac{\varepsilon}{W}\right) & \text { for }|\varepsilon|<W .\end{cases}
$$

In particular, the static limit $\omega=0$ reads

$$
\begin{aligned}
\chi_{s}(q)= & \operatorname{Re}\left[\chi_{F}^{R}(q, \omega=0)\right]=\frac{A}{\pi} \int_{-W}^{\min \left\{W, \epsilon_{F}\right\}} d \varepsilon \rho(\varepsilon) \\
& \times \int_{-k_{F}(\varepsilon)}^{+k_{F}(\varepsilon)} d k g\left(\varepsilon-\frac{\hbar^{2} q^{2}}{2 m_{F}^{*}}-\frac{\hbar^{2} k q}{m_{F}^{*}}\right) .
\end{aligned}
$$

Therefore, it is possible to perform the calculation of the previous expression.

At low frequencies $\left(\hbar \omega \ll \mu_{B}<\epsilon_{F}\right)$, we shall approximate the response function of the Fermi gas by the two first terms in the series about $\omega=0$, i.e.,

$$
\chi_{F}^{R}(q, \omega) \simeq \chi_{s}(q)-i \pi \omega D(q),
$$

where $S(q)=\operatorname{Im}\left[-\chi_{F}^{R}(q)\right] /(\omega \pi)$. Finally, we make use of the spectral properties which relate the retarded response function to its analytical continuation to imaginary frequencies derived in Appendix A:

$$
\chi\left(q, \omega_{n}\right)=-\int \frac{d \omega}{\pi} \frac{\operatorname{Im} \chi(q, \omega)}{i \omega_{n}-\omega} .
$$

In particular, the static limit $\omega_{n}=0$ corresponds to

$$
\chi_{s}(q)=\chi(q, 0)=\int \frac{d \omega}{\pi} \frac{\operatorname{Im} \chi(q, \omega)}{\omega} .
$$

Adding and subtracting the static part, we get

$$
\begin{aligned}
\chi\left(q, \omega_{n}\right) & =\chi_{s}(q)-\int \frac{d \omega}{\pi} \operatorname{Im} \chi(q, \omega)\left[\frac{1}{i \omega_{n}-\omega}+\frac{1}{\omega}\right] \\
& =\chi_{s}(q)-\int \frac{d \omega}{\pi} \frac{\operatorname{Im} \chi(q, \omega)}{\omega}\left[\frac{i \omega_{n}}{i \omega_{n}-\omega}\right],
\end{aligned}
$$

and recall that

$$
\begin{aligned}
\chi(q, \tau)= & \int \frac{d \omega_{n}}{2 \pi} e^{-i \omega_{n} \tau} \chi\left(q, \omega_{n}\right) \\
= & \chi_{s}(q) \delta(\tau)-\int \frac{d \omega}{2 \pi} \frac{\operatorname{Im} \chi(q, \omega)}{\omega} \\
& \times \int_{-\infty}^{+\infty} \frac{d \omega_{n}}{\pi}\left[\frac{i \omega_{n} e^{-i \omega_{n} \tau}}{i \omega_{n}-\omega}\right]
\end{aligned}
$$




$$
\begin{aligned}
= & \chi_{s}(q) \delta(\tau)-\int_{0}^{+\infty} \frac{d \omega}{\pi} \frac{\operatorname{Im} \chi(q, \omega)}{\omega} \\
& \times \int_{-\infty}^{+\infty} \frac{d \omega_{n}}{2 \pi}\left[\frac{i \omega_{n}}{i \omega_{n}-\omega}+\frac{i \omega_{n}}{i \omega_{n}+\omega}\right] e^{-i \omega_{n} \tau} .
\end{aligned}
$$

Thus, upon performing the above integral over $\omega_{n}$ using Cauchy's theorem, the following expression is obtained:

$$
\chi(q, \tau)=\chi_{s}(q) \delta(\tau)+\int_{0}^{+\infty} \frac{d \omega}{\pi} e^{-\omega|\tau|} \operatorname{Im} \chi_{F}^{R}(q, \omega) .
$$

Hence, introducing Eq. (B21) in the expression above,

$$
\chi(q, \tau) \simeq \chi_{s}(q) \delta(\tau)-\frac{D(q)}{\left(|\tau|+\tau_{c}\right)^{2}} .
$$

The first term describes the short-time behavior, which is dominated by screening, whereas the second term describes the long-time behavior, which is dominated by dissipation.

\section{APPENDIX C: RG ANALYSIS AT HALF FILLING}

In order to obtain the RG flow equations, we consider the functional integral representation of the partition function:

$$
Z\left(\tau_{c}\right)=\int[d \phi] e^{-S[\phi]},
$$

where

$$
S[\phi]=S_{0}[\phi]+S_{\mathrm{int}}[\phi],
$$

with $S_{0}[\phi]$ being the Gaussian part of the action [the first term in Eq. (21)]. When writing (C1), we have made explicit the dependence of the partition function on the short-distance cutoff $a_{0} \simeq v \tau_{c}$. Note, however, that the partition function (up to a multiplicative constant) is independent of the cutoff, and we will base our subsequent analysis on this fact. For a general perturbation $S_{\text {int }}[\phi]$, we cannot compute the partition function exactly. Thus, we resort to a perturbative expansion of $Z[(1+$ $\delta \ell) \tau_{c}$ ] (where $\left.\delta \ell \ll 1\right)$ in powers of $S_{\text {int }}$ :

$$
\begin{aligned}
Z\left[(1+\delta \ell) \tau_{c}\right]= & Z_{0}\left[(1+\delta \ell) \tau_{c}\right]\left\{1-\left\langle S_{\text {int }}[\phi]\right\rangle\right. \\
& \left.+\frac{1}{2}\left\langle S_{\text {int }}^{2}[\phi]\right\rangle+\cdots\right\} .
\end{aligned}
$$

To deal with this expansion, it is convenient to define the normal ordered vertex operators,

$$
: e^{2 p i \phi(\mathbf{x})}:=\frac{1}{a_{0}^{p^{2} K}} e^{2 p i \phi(\mathbf{x})},
$$

where $\mathbf{x}=(v \tau, x)$ and the limit $a_{0} \rightarrow 0$ is implicitly understood. Then, when inserting in an expectation value, we have the following operator product expansions (OPEs):

$$
\begin{aligned}
& : e^{2 i p \phi(\mathbf{r})}:: e^{-2 i p \phi\left(\mathbf{r}^{\prime}\right)}: \\
& =\frac{1}{\left|\mathbf{r}-\mathbf{r}^{\prime}\right|^{2 p^{2} K}}:\left[1+2 i p\left(\mathbf{r}-\mathbf{r}^{\prime}\right) \nabla \phi(\mathbf{R})\right. \\
& \left.\quad-2 p^{2}\left[\left(\mathbf{r}-\mathbf{r}^{\prime}\right) \nabla \phi(\mathbf{R})\right]^{2}+\cdots\right]: \\
& \quad \times: e^{2 i p \phi(\mathbf{r})}:: e^{2 i p \phi\left(\mathbf{r}^{\prime}\right)}:=a_{0}^{2 p^{2} K}: e^{4 i p \phi(\mathbf{R})}:+\cdots,
\end{aligned}
$$

where $\mathbf{r}=(v \tau, x), \mathbf{R}=\left(\mathbf{r}-\mathbf{r}^{\prime}\right) / 2, \nabla=[(1 / v) \partial \tau, \partial x]$, and $a_{0}=v \tau_{c}$ is a short-distance cutoff. Next, let us consider the partition function at the scale $(1+\delta l) a_{0}$, where $0<\delta l \ll 1$,

$$
Z\left[(1+\delta l) a_{0}\right]=Z_{0}\left[(1+\delta l) a_{0}\right]\left\{1-\left\langle S_{\mathrm{int}}\right\rangle+\frac{1}{2 !}\left\langle S_{\mathrm{int}}^{2}\right\rangle+\cdots\right\},
$$

where

$$
\begin{gathered}
S_{u}[\phi]=-\frac{g_{u}}{\pi a_{0}^{2-4 K}} \int d x d \tau: \cos 4 \phi(\mathbf{r}) \\
S_{D}[\phi]=-\frac{g_{D}}{a_{0}^{1-2 K}} \int_{\left|\mathbf{r}-\mathbf{r}^{\prime}\right|>a_{0}} d \mathbf{r} d \mathbf{r}^{\prime} \frac{\delta\left(x-x^{\prime}\right)}{\left|\mathbf{r}-\mathbf{r}^{\prime}\right|^{2}}: \cos 2 \phi(\mathbf{r}): \\
\times: \cos 2 \phi\left(\mathbf{r}^{\prime}\right):,
\end{gathered}
$$

and where we have normal ordered the vertex operators.

\section{First-order terms}

Now, let us consider the first-order term $\left\langle S_{\text {int }}\right\rangle=\left\langle S_{u}\right\rangle+$ $\left\langle S_{D}\right\rangle$,

$$
-\left\langle S_{u}\right\rangle=+\frac{g_{u}(l+\delta l)}{\pi\left[(1+\delta l) a_{0}\right]^{2-4 K}} \int d \mathbf{r}\langle: \cos 4 \phi(\mathbf{r}):\rangle .
$$

When comparing with the same operator at the scale $a_{0}$, we find

$$
\begin{aligned}
\frac{g_{u}(l+\delta l)}{[(1+\delta l)]^{2-4 K}} & =g_{u}(l) \Longrightarrow g_{u}(l+\delta l) \\
& =g_{u}(l)[1-(2-4 K) \delta l],
\end{aligned}
$$

which immediately leads to the differential equation

$$
\frac{d g_{u}(l)}{d l}=(2-4 K) g_{u}(l) .
$$

Next, we consider

$$
\begin{aligned}
-\left\langle S_{D}\right\rangle= & +\frac{g_{D}(l+\delta l)}{\pi\left[(1+\delta l) a_{0}\right]^{1-2 K}} \int_{\left|\mathbf{r}-\mathbf{r}^{\prime}\right|>a_{0}(1+\delta l)} d \mathbf{r} d \mathbf{r}^{\prime} \frac{\delta\left(x-x^{\prime}\right)}{\left|\mathbf{r}-\mathbf{r}^{\prime}\right|^{2}} \\
& \times\left\langle: \cos 2 \phi(\mathbf{r}):: \cos 2 \phi\left(\mathbf{r}^{\prime}\right):\right\rangle .
\end{aligned}
$$

To bring this expression to a form which can be compared with the same expression at the cutoff scale $a_{0}$, we first split the integral on $\mathbf{r}$ and $\mathbf{r}^{\prime}$ as follows:

$$
\begin{aligned}
& \int_{\left|\mathbf{r}-\mathbf{r}^{\prime}\right|>a_{0}(1+\delta l)} d \mathbf{r} d \mathbf{r}^{\prime} \ldots \\
& \quad=\int_{\left|\mathbf{r}-\mathbf{r}^{\prime}\right|>a_{0}} d \mathbf{r} d \mathbf{r}^{\prime} \cdots-\int_{a_{0}(1+\delta l)>\left|\mathbf{r}-\mathbf{r}^{\prime}\right|>a_{0}} d \mathbf{r} d \mathbf{r}^{\prime} \ldots
\end{aligned}
$$

Thus, from the first term on the right-hand side of the above equation, we have

$$
\begin{aligned}
& +\frac{g_{D}(l+\delta l)}{\pi\left[(1+\delta l) a_{0}\right]^{1-2 K}} \\
& \quad \times \int_{\left|\mathbf{r}-\mathbf{r}^{\prime}\right|>a_{0}} d \mathbf{r} d \mathbf{r}^{\prime} \frac{\delta\left(x-x^{\prime}\right)}{\left|\mathbf{r}-\mathbf{r}^{\prime}\right|^{2}}\left\langle: \cos 2 \phi(\mathbf{r}):: \cos 2 \phi\left(\mathbf{r}^{\prime}\right):\right\rangle .
\end{aligned}
$$

Hence, following the same procedure as before,

$$
\frac{g_{D}(l+\delta l)}{[(1+\delta l)]^{1-2 K}}=g_{D}(l) \Longrightarrow \frac{d g_{D}(l)}{d l}=(1-2 K) g_{D}(l) \text {. }
$$


Next, we take up the contribution from the second term in Eq. (C13):

$$
\begin{aligned}
- & \frac{g_{D}(l+\delta l)}{a_{0}^{1-2 K}} \int_{a_{0}(1+\delta l)>\left|\mathbf{r}-\mathbf{r}^{\prime}\right|>a_{0}} d \mathbf{r} d \mathbf{r}^{\prime} \frac{\delta\left(x-x^{\prime}\right)}{\left|\mathbf{r}-\mathbf{r}^{\prime}\right|^{2}} \\
& \quad \times\left\langle: \cos 2 \phi(\mathbf{r}):: \cos 2 \phi\left(\mathbf{r}^{\prime}\right):\right\rangle \\
= & -\frac{g_{D}(l+\delta l)}{2 a_{0}^{1-2 K}} \int_{a_{0}(1+\delta l)>\left|\mathbf{r}-\mathbf{r}^{\prime}\right|>a_{0}} d \mathbf{r} d \mathbf{r}^{\prime} \frac{\delta\left(x-x^{\prime}\right)}{\left|\mathbf{r}-\mathbf{r}^{\prime}\right|^{2+2 K}} \\
& \times\left\langle:\left\{\left[1-2\left[\left(\mathbf{r}-\mathbf{r}^{\prime}\right) \nabla \phi(\mathbf{R})\right]^{2}+\cdots\right\}:\right\rangle\right. \\
= & -\frac{g_{D}(l+\delta l)}{2 a_{0}^{1-2 K}} \int_{a_{0}(1+\delta l)>\left|\mathbf{r}-\mathbf{r}^{\prime}\right|>a_{0}} d \mathbf{r} d \mathbf{r}^{\prime} \frac{\delta\left(x-x^{\prime}\right)}{\left|\mathbf{r}-\mathbf{r}^{\prime}\right|^{2}} \\
& \times d a_{0}^{2 k}\langle: \cos 4 \phi(\mathbf{R}):\rangle .
\end{aligned}
$$

Introducing $\mathbf{u}=\mathbf{r}-\mathbf{r}^{\prime}$ leads to

$$
\begin{gathered}
-\frac{g_{D}(l) \delta l}{a_{0}^{1-4 K}} \int_{a_{0}(1+\delta l)>|\mathbf{u}|>a_{0}} d \mathbf{u} \frac{\delta\left(u_{x}\right)}{|\mathbf{u}|^{2}} \\
=\left[\int_{a_{0}}^{a_{0}(1+\delta l)} \frac{d u_{x}}{u_{x}^{2}}\right] \frac{1}{a_{0}^{1-4 K}}=\frac{\delta l}{a_{0}^{2-4 K}} .
\end{gathered}
$$

Hence, the second term in Eq. (C16) yields

$$
-\frac{g_{D}(l) \delta l}{a_{0}^{2-4 K}} \int d \mathbf{r}: \cos 4 \phi(\mathbf{r}): .
$$

Therefore, the flow equation for $g_{u}(l)$ [i.e., Eq. (C11)] must be modified to

$$
\begin{aligned}
\frac{g_{u}(l+\delta l)}{[(1+\delta l)]^{2-4 K}-\pi g_{D}(l) \delta l=} & g_{u}(l), \\
g_{u}(l+\delta l)= & {[1+(2-4 K) \delta l] g_{u}(l) } \\
& +\pi g_{D}(l) \delta l, \\
\frac{d g_{u}(l)}{d l}= & (2-4 K) g_{u}(l)-\pi g_{D}(l) .
\end{aligned}
$$

Finally, it is necessary to consider the first term in Eq. (C16). To this end, we need to consider the following integral with $\mathbf{r}-\mathbf{r}^{\prime}=\mathbf{u}=\left(u_{\tau}, u_{x}\right)$ :

$$
\begin{gathered}
-\frac{g_{D}(l) \delta l}{a_{0}^{1-2 K}} \int_{a_{0}(1+\delta l)>|\mathbf{u}|>a_{0}} d \mathbf{u} \frac{\delta\left(u_{x}\right)}{|\mathbf{u}|^{2+2 K}} \\
=\left[\int_{a_{0}}^{a_{0}(1+\delta l)} \frac{d u}{u^{2 K}}\right] \frac{1}{a_{0}^{1-4 K}} \\
=\left[\int_{a_{0}}^{a_{0}(1+\delta l)} d\left(\frac{u}{a_{0}}\right)\left(\frac{a_{0}}{u}\right)^{2 K}\right]=\delta l .
\end{gathered}
$$

Thus, a term of the following form is generated:

$$
\frac{2 g_{D}(l)}{v(l)} \delta l \int d x d \tau\left\langle\left(\partial_{\tau} \phi\right)^{2}\right\rangle .
$$

Note that this term has the same form of the $\frac{1}{2 \pi K v}\left(\partial_{\tau} \phi\right)^{2}$ operator in the Gaussian action, $S_{0}[\phi]$. When reexponentiated, we find, upon comparing with the same action at the cutoff scale,

$$
\frac{1}{2 \pi K(l+\delta l) v(l+\delta l)}-2 \frac{g_{D}(l)}{v(l)} \delta l=\frac{1}{2 \pi K(l) v(l)} .
$$

Hence,

$$
\begin{aligned}
\frac{1}{K(l+\delta l) v(l+\delta l)} & =\frac{1}{2 \pi K(l) v(l)}+4 \pi \frac{g_{D}(l)}{v(l)} \delta l \\
& \Longrightarrow \frac{d}{d l}\left(\frac{1}{K v}\right)=\frac{4 \pi g_{D}}{v}
\end{aligned}
$$

Furthermore, the coefficient of $\int d x d \tau\left(\partial_{x} \phi\right)^{2}$ is not renormalized,

$$
\frac{v(l+\delta l)}{K(l+\delta l)}=\frac{v(l)}{K(l)} \Longrightarrow \frac{d}{d l}\left(\frac{v}{K}\right)=0 .
$$

From these equations, we can extract the RG flow equations for $K$ and $v$ :

$$
\begin{aligned}
\frac{1}{K} \frac{d v}{d l} & +v \frac{d}{d l}\left(\frac{1}{K}\right)=0, \\
\frac{1}{K v^{2}} \frac{d v}{d l}+\frac{1}{v} \frac{d}{d l}\left(\frac{1}{K}\right) & =\frac{4 \pi g_{D}}{v} \Longrightarrow \frac{1}{K} \frac{d v}{d l}+v \frac{d}{d l}\left(\frac{1}{K}\right) \\
& =4 \pi g_{D} v .
\end{aligned}
$$

Thus, adding Eqs. (C25) and (C26), we find

$$
2 \frac{d}{d l}\left(\frac{1}{K}\right)=4 \pi g_{D} \Longrightarrow \frac{d}{d l}\left(\frac{1}{K}\right)=2 \pi g_{D} .
$$

\section{Second-order terms}

After considering the first-order contributions, we need to take up the second order,

$$
\frac{1}{2 !}\left\langle S_{\text {int }}^{2}\right\rangle=\frac{1}{2 !}\left\langle S_{u}^{2}[\phi]\right\rangle+\cdots .
$$

We do not consider terms of order $g_{u} g_{D}$ or $g_{D}^{2}$ because $g_{D} \propto$ $g_{B F}^{2}$ is already second order and $g_{u} \ll 1$ is considered small. Thus, we take

$$
\begin{aligned}
\frac{1}{2 !}\left\langle S_{u}^{2}[\phi]\right\rangle= & \frac{1}{2}\left\{\frac{g_{u}(l+\delta l)}{\pi\left[(1+\delta l) a_{0}\right]^{2-4 K}}\right\}^{2} \int_{\left|\mathbf{r}-\mathbf{r}^{\prime}\right|>a_{0}(1+\delta l)} d \mathbf{r} d \mathbf{r}^{\prime} \\
& \times\left\langle: \cos 4 \phi(\mathbf{r}):: \cos 4 \phi\left(\mathbf{r}^{\prime}\right):\right\rangle .
\end{aligned}
$$

Again, we split the integral as in Eq. (C13), which leads to

$$
\begin{aligned}
- & \frac{1}{2}\left(\frac{g_{u}(l)}{\pi a_{0}^{2-4 K}}\right)^{2} \int_{a_{0}(1+\delta l)>\left|\mathbf{r}-\mathbf{r}^{\prime}\right|>a_{0}} \frac{1}{2} d \mathbf{r} d \mathbf{r}^{\prime} \\
& \times\left\langle:\left\{1-8\left[\left(\mathbf{r}-\mathbf{r}^{\prime}\right) \nabla \phi(\mathbf{R})\right]^{2}+\cdots\right\}:\right\rangle \frac{1}{\left|\mathbf{r}-\mathbf{r}^{\prime}\right|^{8 K-4}} \\
= & \frac{4}{\pi^{2}} \int d \mathbf{R}\left\langle\left|\nabla_{\mathbf{R}} \phi(\mathbf{R})\right|^{2}\right\rangle \\
& \times \int_{a_{0}(1+\delta l)>\left|\mathbf{r}-\mathbf{r}^{\prime}\right|>a_{0}}\left(\frac{a_{0}}{|\mathbf{u}|}\right)^{8 K-4} \cos ^{2} \phi+\cdots \\
= & \frac{2}{\pi} g_{u}^{2} \delta l \int d \mathbf{r}\left\langle:[\nabla \phi(\mathbf{r})]^{2}:\right\rangle+\cdots
\end{aligned}
$$

Thus, we need to revise our previously derived equations for the renormalization of the Gaussian action 
parameters,

$$
\frac{1}{2 \pi K(l+\delta l) v(l+\delta l)}-2 \frac{g_{D}(l)}{v(l)} \delta l-2 \frac{g_{u}^{2}(l)}{\pi v(l)} \delta l=\frac{1}{2 \pi K(l) v(l)},
$$

$$
\frac{v(l+\delta l)}{2 \pi K(l+\delta l)}-\frac{2 v(l) g_{u}^{2}(l) \delta l}{\pi}=\frac{v(l)}{2 \pi K(l)} .
$$

Hence,

$$
\begin{gathered}
\frac{d}{d l}\left(\frac{1}{K v}\right)=\frac{4 \pi g_{D}}{v}+4 \pi \frac{g_{u}^{2}}{v}, \\
\frac{d}{d l}\left(\frac{v}{K}\right)=4 v g_{u}^{2}, \\
\frac{1}{v} \frac{d}{d l}\left(\frac{1}{K}\right)-\frac{1}{K v^{2}} \frac{d v}{d l}=\frac{4 \pi}{v}\left[g_{D}+\frac{g_{u}^{2}}{\pi}\right], \\
v \frac{d}{d l}\left(\frac{1}{K}\right)+\frac{1}{K} \frac{d v}{d l}=4 v g_{u}^{2} .
\end{gathered}
$$

Hence, the RG flow equations for both $K$ and $v$,

$$
\begin{gathered}
\frac{d}{d l}\left(\frac{1}{K}\right)-\frac{1}{K v^{2}} \frac{d v}{d l}=\frac{4 \pi}{v}\left[g_{D}+\frac{g_{u}^{2}}{\pi}\right], \\
\frac{d}{d l} v=-2 \pi g_{D} K v .
\end{gathered}
$$

Finally, in the analysis of the second-order contributions, we need to consider the term

$$
\begin{aligned}
O\left(g_{u} g_{D}\right)= & \frac{2 g_{u}(l+\delta l)}{\pi\left[a_{0}(1+\delta l)\right]^{4-2 K}} \frac{g_{D}(l+\delta l)}{\left[a_{0}(1+\delta l)\right]^{1-2 K}} \\
& \times \int_{*} d \mathbf{r}_{1} d \mathbf{r}_{2} d \mathbf{r}_{3} \frac{\delta\left(x_{1}-x_{2}\right)}{\left|\mathbf{r}_{1}-\mathbf{r}_{2}\right|^{2}} \\
& \times\left\langle: \cos 4 \phi\left(\mathbf{r}_{1}\right) \cos 4 \phi\left(\mathbf{r}_{2}\right) \cos 4 \phi\left(\mathbf{r}_{3}\right):\right\rangle,
\end{aligned}
$$

where the star $(*)$ under the integral means that $\left|\mathbf{r}_{1}-\mathbf{r}_{2}\right|>$ $a_{0}(1+\delta l),\left|\mathbf{r}_{1}-\mathbf{r}_{3}\right|>a_{0}(1+\delta l)$, and $\left|\mathbf{r}_{2}-\mathbf{r}_{3}\right|>a_{0}(1+\delta l)$. Let us consider the contribution resulting from the OPE when $\mathbf{r}_{1} \rightarrow \mathbf{r}_{2}$ (or, equivalently, $\mathbf{r}_{1} \rightarrow \mathbf{r}_{3}$ ),

$: \cos 4 \phi\left(\mathbf{r}_{1}\right):: \cos 4 \phi\left(\mathbf{r}_{2}\right):=\frac{1}{2\left|\mathbf{r}-\mathbf{r}^{\prime}\right|^{4 K}}: \cos 2 \phi(\mathbf{R}):+\cdots$.

(C40)
Hence, as the above factor of 2 is canceled by the two possible contractions $\mathbf{r}_{1} \rightarrow \mathbf{r}_{2}$ and $\mathbf{r}_{2} \rightarrow \mathbf{r}_{3}$, we find

$$
\begin{aligned}
O\left(g_{u} g_{D}\right)= & -\frac{2 g_{u}(l+\delta l)}{\pi\left[a_{0}(1+\delta l)\right]^{4-2 K}} \frac{g_{D}(l+\delta l)}{\left[a_{0}(1+\delta l)\right]^{1-2 K}} \\
& \times \int_{a_{0}(1+\delta l)>|\rho|>a_{0}} d \rho \frac{1}{|\rho|^{4 K}} \int d \mathbf{R} d \mathbf{r}_{3} \frac{\delta\left(X-x_{3}\right)}{\left|\mathbf{R}-\mathbf{r}_{3}\right|^{2}} \\
& \times\left\langle: \cos 4 \phi(\mathbf{R}) \cos 4 \phi\left(\mathbf{r}_{3}\right):\right\rangle+\cdots \\
= & -\frac{4 g_{u}(l) g_{D}(l)}{\pi\left[a_{0}(1+\delta l)\right]^{1-2 K}} \delta l \\
& \times \int d \mathbf{r} d \mathbf{r}^{\prime} \frac{\delta\left(x-x^{\prime}\right)}{\left|\mathbf{r}-\mathbf{r}^{\prime}\right|^{2}}\left\langle: \cos 2 \phi(\mathbf{r}):: \cos 2 \phi\left(\mathbf{r}^{\prime}\right):\right\rangle .
\end{aligned}
$$

Therefore, we obtain the following differential equation:

$$
\begin{aligned}
& \frac{g_{D}(l+\delta l)}{\left[a_{0}(1+\delta l)\right]^{1-2 K}}-\frac{4 g_{u}(l) g_{D}(l) \delta l}{\left[a_{0}(1+\delta l)\right]^{1-2 K}}=\frac{g_{D}(l)}{a_{0}^{1-2 K}} \Longrightarrow \frac{g_{D}}{d l} \\
& =(1-2 K) g_{D}+4 g_{D} g_{u} .
\end{aligned}
$$

Thus, the complete set of RG flow equations reads

$$
\begin{gathered}
\frac{d v}{d l}=-4 \pi g_{D} K v, \\
\frac{d K}{d l}=-\left(4 g_{u}^{2}+2 \pi g_{D}\right) K^{2}, \\
\frac{d g_{u}}{d l}=(2-4 K) g_{u}+\pi g_{D}, \\
\frac{d g_{D}}{d l}=(1-2 K) g_{D}+4 g_{D} g_{u} .
\end{gathered}
$$

\section{APPENDIX D: SCHA}

We have adopted a variational self-consistent harmonic approximation (SCHA) by choosing a trial effective action such as in Eq. (50). To find the variational estimate of the free energy, we have to perform the averages of the effective action with respect to the trial effective action, by using $S_{0}$ [Eq. (21)], $S_{u}$ [Eq. (22)] with $p=2$ for half-lattice filling, $S_{D}^{b}$ [Eq. (36)], and $S_{D}^{u}$ [Eq. (37)]. Thus, the variational free energy $F_{\text {var }}$ that follows from Eq. (51) will be

$$
\begin{aligned}
F_{\mathrm{var}}\left[G_{v}\right]= & -\frac{T}{2} \int \frac{d q d \omega}{(2 \pi)^{2}} \ln G_{v}(q, \omega)+T\left[\frac{1}{2 \pi K}\left(\frac{\omega^{2}}{v_{s}}+v_{s} q^{2}\right)\right] G_{v}(q, \omega)-T \frac{g_{u}}{a_{0} \tau_{0}} \int d x d \tau e^{-8 \int \frac{d q d \omega}{(2 \pi)^{2}} G_{v}(q, \omega)} \\
& -T \frac{g_{D}}{a_{0}} \int d x \int_{\left|\tau-\tau^{\prime}\right|>\tau_{0}} d \tau d \tau^{\prime} \frac{e^{-4 \int \frac{d q d \omega}{2 \pi)^{2}}\left[1-\cos \omega\left(\tau-\tau^{\prime}\right)\right] G_{v}(q, \omega)}}{\left|\tau-\tau^{\prime}\right|^{2}} \\
& -T \frac{g_{D}}{a_{0}} \int d x \int_{\left|\tau-\tau^{\prime}\right|>\tau_{0}} d \tau d \tau^{e^{\prime}} \frac{e^{-4 \int \frac{d q d \omega}{(2 \pi)^{2}}\left[1+\cos \omega\left(\tau-\tau^{\prime}\right)\right] G_{v}(q, \omega)}}{\left|\tau-\tau^{\prime}\right|^{2}}-\left\langle S_{v}\right\rangle_{v} .
\end{aligned}
$$

Therefore, requiring $\delta F_{\mathrm{var}}\left[G_{v}\right] / \delta G_{v}=0$ yields

$$
\begin{aligned}
\delta F_{\mathrm{var}}\left[G_{v}\right] / \delta G_{v}= & -\frac{T}{2} \int \frac{d q d \omega}{(2 \pi)^{2}} \frac{1}{G_{v}(q, \omega)}+T\left[\frac{1}{2 \pi K}\left(\frac{\omega^{2}}{v_{s}}+v_{s} q^{2}\right)\right]+T \frac{8 g_{u}}{(2 \pi)^{2} a_{0} \tau_{0}} \alpha^{2}(\eta, \Delta, K) \\
& +T \frac{4 g_{D}}{(2 \pi)^{2} a_{0}}\left[\alpha(\eta, \Delta, K)+\alpha^{2}(\eta, \Delta, K)\right]=0,
\end{aligned}
$$

where $\alpha(\eta, \Delta, K)=\left[\frac{\eta K \pi+2 \sqrt{K \pi \Delta}}{4}\right]^{2 K}$. Keeping the $\tau$-independent terms in the integrals in Eq. (D1), which yield the leading contributions in $\omega$ to $G_{v}(q, \omega)$, leads to Eqs. (52) and (53). 
[1] G. Baym and C. J. Pethick, Landau Fermi Liquid Theory (Wiley, New York, 1991).

[2] A. A. Abrikosov, L. P. Gorkov, and I. E. Dzyaloshinski, Methods of Quantum Field Theory in Statistical Physics (Dover, New York, 1975).

[3] I. Bloch, J. Dalibard, and W. Zwerger, Rev. Mod. Phys. 80, 885 (2008).

[4] T. L. Ho, Phys. Rev. Lett. 81, 742 (1998); K. Molmer, ibid. 80, 1804 (1998); L. Viverit, C. J. Pethick, and H. Smith, Phys. Rev. A 61, 053605 (2000); A. P. Albus, S. A. Gardiner, F. Illuminati, and M. Wilkens, ibid. 65, 053607 (2002).

[5] G. Roati, F. Riboli, G. Modugno, and M. Inguscio, Phys. Rev. Lett. 89, 150403 (2002).

[6] M. Zaccanti, C. D’Errico, F. Ferlaino, G. Roati, M. Inguscio, and G. Modugno, Phys. Rev. A 74, 041605(R) (2006).

[7] K. Günter, T. Stöferle, H. Moritz, M. Köhl, and T. Esslinger, Phys. Rev. Lett. 96, 180402 (2006).

[8] S. Ospelkaus, C. Ospelkaus, O. Wille, M. Succo, P. Ernst, K. Sengstock, and K. Bongs, Phys. Rev. Lett. 96, 180403 (2006).

[9] D.-S. Lühmann, K. Bongs, K. Sengstock, and D. Pfannkuche, Phys. Rev. Lett. 101, 050402 (2008).

[10] Th. Best, S. Will, U. Schneider, L. Hackermüller, D. van Oosten, I. Bloch, and D.-S. Lühmann, Phys. Rev. Lett. 102, 030408 (2009).

[11] S. Sugawa, K. Inaba, S. Taie, R. Yamazaki, M. Yamashita, and Y. Takahashi, Nat. Phys. 7, 642 (2011).

[12] G. Lamporesi, J. Catani, G. Barontini, Y. Nishida, M. Inguscio, and F. Minardi, Phys. Rev. Lett. 104, 153202 (2010).

[13] M. K. Tey, S. Stellmer, R. Grimm, and F. Schreck, Phys. Rev. A 82, 011608(R) (2010).

[14] J. W. Park, C.-H. Wu, I. Santiago, T. G. Tiecke, S. Will, P. Ahmadi, and M. W. Zwierlein, Phys. Rev. A 85, 051602(R) (2012); 84, 011601(R) (2011).

[15] J. Heinze, S. Götze, J. S. Krauser, B. Hundt, N. Fläschner, D.-S. Lühmann, C. Becker, and K. Sengstock, Phys. Rev. Lett. 107, 135303 (2011).

[16] H. Hara, Y. Takasu, Y. Yamaoka, J. M. Doyle, and Y. Takahashi, Phys. Rev. Lett. 106, 205304 (2011).

[17] K. K. Das, Phys. Rev. Lett. 90, 170403 (2003).

[18] L. Pollet, C. Kollath, U. Schollwöck, and M. Troyer, Phys. Rev. A 77, 023608 (2008).

[19] M. A. Cazalilla and A. F. Ho, Phys. Rev. Lett. 91, 150403 (2003).

[20] L. Mathey, D.-W. Wang, W. Hofstetter, M. D. Lukin, and E. Demler, Phys. Rev. Lett. 93, 120404 (2004).

[21] B. Wang, D.-W. Wang, and S. Das Sarma, Phys. Rev. A 82, 021602(R) (2010).

[22] E. Taylor, A. Griffin, and Y. Ohashi, Phys. Rev. A 76, 023614 (2007).

[23] F. M. Marchetti, C. J. M. Mathy, D. A. Huse, and M. M. Parish, Phys. Rev. B 78, 134517 (2008).

[24] G. Refael and E. Demler, Phys. Rev. B 77, 144511 (2008).

[25] R. M. Lutchyn, S. Tewari, and S. DasSarma, Phys. Rev. B 78, 220504 (2008).

[26] M. Rizzi and A. Imambekov, Phys. Rev. A 77, 023621 (2008).

[27] F. M. Marchetti, Th. Jolicoeur, and M. M. Parish, Phys. Rev. Lett. 103, 105304 (2009).
[28] S. Modak, S.-W. Tsai, and K. Sengupta, Phys. Rev. B 84, 134508 (2011).

[29] F. Hebert, G. G. Batrouni, X. Roy, and V. G. Rousseau, Phys. Rev. B 78, 184505 (2008).

[30] K. Noda, R. Peters, N. Kawakami, and T. Pruschke, Phys. Rev. A 85, 043628 (2012).

[31] P. Anders, P. Werner, M. Troyer, M. Sigrist, and Lode Pollet, Phys. Rev. Lett. 109, 206401 (2012).

[32] E. Malatsetxebarria, F. Marchetti, and M. A. Cazalilla, Phys. Rev. A. 88, 033604 (2013).

[33] E. Fratini and P. Pieri, Phys. Rev. A 85, 063618 (2012).

[34] X. Yin, X.-W. Guan, Y. Zhang, and S. Chen, Phys. Rev. A 85, 013608 (2012); X.-W. Guan, M. T. Batchelor, and J.-Y. Lee, ibid. 78, 023621 (2008).

[35] M. Snoek, I. Titvinidze, I. Bloch, and W. Hofstetter, Phys. Rev. Lett. 106, 155301 (2011).

[36] T. Enss and W. Zwerger, Eur. Phys. J. B 68, 383 (2009).

[37] Kun Yang, Phys. Rev. B 77, 085115 (2008).

[38] M. A. Cazalilla, R. Citro, T. Giamarchi, E. Orignac, and M. Rigol, Rev. Mod. Phys. 83, 1405 (2011).

[39] T. Giamarchi, Quantum Physics in One Dimension (Clarendon, Oxford, U.K., 2004).

[40] F. D. M. Haldane, Phys. Rev. Lett. 45, 1359 (1980); 47, 1840 (1981); 48, 569(E) (1982).

[41] E. Haller, R. Hart, M. J. Mark, J. G. Danzl, L. Reichsllner, M. Gustavsson, M. Dalmonte, G. Pupillo, and H.-C. Nägerl, Nature (London) 466, 597 (2010).

[42] A. O. Gogolin, A. A. Nersesyan, and A. M. Tsvelik, Bosonization and Strongly Correlated Systems (Cambridge University Press, Cambridge, U.K., 1998).

[43] E. H. Lieb and W. Liniger, Phys. Rev. 130, 1605 (1963); E. H. Lieb, ibid. 130, 1616 (1963).

[44] M. Olshanii, Phys. Rev. Lett. 81, 938 (1998).

[45] R. Citro, E. Orignac, S. De Palo, and M. L. Chiofalo, Phys. Rev. A 75, 051602 (2007); P. Pedri, S. De Palo, E. Orignac, R. Citro, and M. L. Chiofalo, ibid. 77, 015601 (2008); S. De Palo, E. Orignac, R. Citro, and M. L. Chiofalo, Phys. Rev. B 77, 212101 (2008).

[46] M. Valiente, Europhys. Lett. 98, 10010 (2012); M.-T. Batchelor, M. Bortz, X.-W. Guan, and N. Oelkers, J. Stat. Mech. (2005) L10001; S. Chen, Xi-Wen Guan, X.-G. Yin, L. Guan, and M. T. Batchelor, Phys. Rev. A 81, 031608(R) (2010); R. Qi and X.-W. Guan, Europhys. Lett. 101, 40002 (2013).

[47] E. Haller, M. Gustavsson, M. J. Mark, J. G. Danzl, R. Hart, G. Pupillo, and H.-C. Nägerl, Science 325, 1224 (2009).

[48] M. A. Cazalilla, F. Sols, and F. Guinea, Phys. Rev. Lett. 97, 076401 (2006).

[49] T. Stöferle, H. Moritz, C. Schori, M. Köhl, and T. Esslinger, Phys. Rev. Lett. 92, 130403 (2004).

[50] A. Iucci, M. A. Cazalilla, A. F. Ho, and T. Giamarchi, Phys. Rev. A 73, 041608(R) (2006).

[51] C. Kollath, A. Iucci, T. Giamarchi, W. Hofstetter, and U. Schollwöck, Phys. Rev. Lett. 97, 050402 (2006).

[52] M. D. Girardeau, J. Math. Phys. 1, 516 (1960).

[53] N. Economou, Green's Functions in Quantum Physics, 3rd ed. (Springer-Verlag, Berlin-Heilderberg, 2006), p. 89. 